Publ. RIMS, Kyoto Univ.

19 (1983), 841-886

\title{
The Diffusion Approximation of the Boltzmann Equation of Maxwellian Molecules ${ }^{\dagger}$
}

\author{
By \\ Tadahisa FUNAKI*
}

\section{$\S 1$. Introduction}

L. D. Landau studied a so-called weakly coupled gas to analyze the time evolution of a plasma and derived an equation for the one particle density from the Boltzmann equation of Coulomb molecules (see Balescu [1] and also Lifshitz and Pitaevskii [12]). Inspired by his work, in this paper, we shall discuss the diffusion approximation of the Boltzmann equation of spatially homogeneous Maxwellian molecules by applying probabilistic methods.

Landau saw through the importance of distant encounters for the charged particles. At large distances, the particles are deflected with only a slight change in their velocities. This suggests that the collisions except for small changes in velocities may be neglected. Accordingly, he derived a nonlinear diffusion equation which is called the Landau equation now. An infinite gas of molecules with the rather idealized type of interaction described above is called a weakly coupled gas. Spohn [17] discussed these matters from the mathematical view point.

The Boltzmann equation governs the time evolution of the one particle density $u(t, x),(t, x) \in(0, \infty) \times \boldsymbol{R}^{3}$, of a dilute gas where $x$ is the velocity of the particle. In the case of spatially homogeneous Maxwellian molecules, the equation has a simple form:

Communicated by S. Matsuura, November 25, 1982.

* Department of Mathematics, Faculty of Science, Nagoya University, Nagoya 464, Japan.

† Supported in part by the Sakkokai Foundation. 


$$
\begin{aligned}
\frac{\partial u}{\partial t}(t, x)= & \int_{(0, \pi) \times(0,2 \pi) \times \boldsymbol{R}^{3}}\left\{u\left(t, x^{*}\right) u\left(t, y^{*}\right)\right. \\
& -u(t, x) u(t, y)\} Q(d \theta) d \phi d y,(t, x) \in(0, \infty) \times \boldsymbol{R}^{3},
\end{aligned}
$$

where $Q$ is a measure on $(0, \pi)$ which characterizes the collision of particles and satisfies $\int_{0}^{\pi} \theta Q(d \theta)<\infty$. The two values $x^{*}$ and $y^{*}$ stand for the velocities of particles after collision and are given by the following formula:

$$
\left\{\begin{array}{r}
x^{*}=x \cos ^{2} \frac{\theta}{2}+y \sin ^{2} \frac{\theta}{2}+\frac{1}{2} e(\phi)|x-y| \sin \theta \\
y^{*}=x \sin ^{2} \frac{\theta}{2}+y \cos ^{2} \frac{\theta}{2}-\frac{1}{2} e(\phi)|x-y| \sin \theta, \\
(x, y, \theta, \phi) \in \boldsymbol{R}^{3} \times \boldsymbol{R}^{3} \times(0, \pi) \times(0,2 \pi),
\end{array}\right.
$$

where $e(\phi)=e(\phi ; x, y) \in \mathbb{R}^{3}, \phi \in(0,2 \pi)$, are unit vectors which are perpendicular to $x-y$ and satisfy that

$$
\begin{aligned}
& |x-y| \frac{d e}{d \phi}(\phi)=(x-y) \times e(\phi) \\
& \quad(=\text { outer product of } x-y \text { and } e(\phi)), \phi \in(0,2 \pi) .
\end{aligned}
$$

We consider, instead of (1.1), its weak version:

$$
\begin{aligned}
& \frac{d}{d t}\langle u(t), \phi\rangle \\
= & \int_{(0, \pi) \times \boldsymbol{R}^{3} \times \boldsymbol{R}^{3}} G(x, y, \theta ; \psi) Q(d \theta) u(t, d x) u(t, d y), \psi \in C_{0}^{\infty}\left(R^{3}\right), t \geqq 0,
\end{aligned}
$$

where $C_{0}^{\infty}\left(R^{3}\right)$ is the space of real valued $C^{\infty}$-functions on $\mathbb{R}^{3}$ with compact supports,

$$
G(x, y, \theta ; \psi)=\int_{0}^{2 \pi}\left\{\psi\left(x^{*}\right)-\psi(x)\right\} d \phi
$$

and $\langle u(t), \psi\rangle$ represents the integral of $\psi$ with respect to a probability measure solution $u(t) \equiv u(t, d x)$. Denote by $\mathscr{L}_{2}$ the space of probability distributions $f$ on $\mathbb{R}^{3}$ satisfying $\int_{R^{3}}|x|^{2} f(d x)<\infty$. Then probabilistic methods guarantee the solvability of the equation (1.3) with an initial value $f \in \mathscr{L}_{2}$ (see Section 2 and Tanaka [20], [21]). The solution $u(t)$ $\equiv T_{t} f$ defines a nonlinear semigroup on the space $\mathscr{L}_{2}$. The operator $T_{t}$ 
is determined by the measure $Q$ so that we denote it by $T_{t}(Q)$.

After the idea of Landau, for $\varepsilon ; 0<\varepsilon<1$, we consider transformations of the time $t$ and the measure $Q: t \rightarrow \varepsilon^{-2} t$ and $Q \rightarrow Q_{\varepsilon}$, where $Q_{\varepsilon}$ is a measure on $(0, \pi)$ defined by $Q_{\varepsilon}(B)=Q\left(\varepsilon^{-1} B \cap(0, \pi)\right)$ for every Borel subset $B$ of $(0, \pi)$. According to the transformation, we have a nonlinear semigroup $\left\{T_{t}^{\varepsilon} ; t \geqq 0\right\}$ on the space $\mathscr{L}_{2}$ defined by $T_{t}^{\varepsilon}=T_{\varepsilon-2 t}\left(Q_{\varepsilon}\right)$ and the Boltzmann equation turns into

$$
\begin{aligned}
& \frac{d}{d t}\left\langle u^{\varepsilon}(t), \psi\right\rangle=\varepsilon^{-2} \int_{(0, \pi) \times \boldsymbol{R}^{3} \times \mathbb{R}^{3}} G(x, y, \theta ; \psi) \\
& \times Q_{\varepsilon}(d \theta) u^{\varepsilon}(t, d x) u^{\varepsilon}(t, d y), \psi \in C_{0}^{\infty}\left(\mathbb{R}^{3}\right), t \geqq 0,
\end{aligned}
$$

where $u^{\varepsilon}(t)=T_{t}^{\varepsilon} f$. We note that, as $\varepsilon \rightarrow 0$, the collisions per unit time interval increase, but, on the other hand, the change of the velocity of a particle in a collision becomes small. The problem is to determine an equation which governs the time evolution of the $\operatorname{limit}: \lim _{\varepsilon \downarrow 0} T_{t}^{\varepsilon} f$, $f \in \mathscr{Q}_{2}$.

We now introduce the Landau equation of spatially homogeneous Maxwellian molecules:

$$
\begin{aligned}
& \frac{\partial u}{\partial t}(t, x)=\frac{1}{2} \sum_{i, j=1}^{3} \frac{\partial^{2}}{\partial x_{i} \partial x_{j}}\left\{\boldsymbol{a}_{i j}(x, u(t)) u(t, x)\right\} \\
& \quad-\sum_{i=1}^{3} \frac{\partial}{\partial x_{i}}\left\{\boldsymbol{b}_{i}(x, u(t)) u(t, x)\right\},(t, x) \in(0, \infty) \times \mathbb{R}^{3}, x=\left(x_{i}\right)_{i=1}^{3},
\end{aligned}
$$

where $u(t)$ is a probability distribution on $\mathbb{R}^{3}$ with density $u(t, x)$ relative to the Lebesgue measure $d x$ and where

$$
\begin{aligned}
\boldsymbol{a}(x, u) & =\left\{\boldsymbol{a}_{i j}(x, u)\right\}_{i, j=1}^{3}=\int_{\mathbb{R}^{3}} \boldsymbol{a}(x-y) u(d y), \\
\boldsymbol{a}(x) & =\left\{\boldsymbol{a}_{i j}(x)\right\}_{i, j=1}^{3}=\left\{\alpha\left(\delta_{i j}|x|^{2}-x_{i} x_{j}\right)\right\}_{i, j=1}^{3}, \\
\boldsymbol{b}(x, u) & =\left\{\boldsymbol{b}_{i}(x, u)\right\}_{i=1}^{3}=\int_{\mathbb{R}^{3}} \boldsymbol{b}(x-y) u(d y), \\
\boldsymbol{b}(x) & =\left\{\boldsymbol{b}_{i}(x)\right\}_{i=1}^{3}=-2 \alpha x,(x, u) \in \mathbb{R}^{3} \times \mathscr{L}_{2},
\end{aligned}
$$

with some positive $\alpha$. This is a kind of nonlinear diffusion equation and its weak version is given by

$$
\frac{d}{d t}\langle u(t), \psi\rangle=\left\langle u(t), \mathcal{A}_{u(t)} \psi\right\rangle, \psi \in C_{0}^{\infty}\left(\boldsymbol{R}^{3}\right), t \geqq 0,
$$


where

$$
\mathcal{A}_{u}=\frac{1}{2} \sum_{i, j=1}^{3} \boldsymbol{a}_{i j}(x, u) \frac{\partial^{2}}{\partial x_{i} \partial x_{j}}+\sum_{i=1}^{3} \boldsymbol{b}_{i}(x, u) \frac{\partial}{\partial x_{i}}, u \in \mathscr{L}_{2} .
$$

Similarly to the case of the Boltzmann equation, we can solve the equation (1.6) with an initial value $f \in \mathscr{L}_{2}$ by using probabilistic methods. The solution $u(t) \equiv U_{t} f$ defines a nonlinear semigroup on the space $\mathscr{L}_{2}$.

The main object of this paper is to show that $U_{t} f=\lim _{\varepsilon \downarrow 0} T_{t}^{\varepsilon} f$ holds for $f \in \mathscr{Q}_{2}$ where $\left\{U_{t}\right\}$ is the nonlinear semigroup defined by (1.6) with $\alpha=\frac{\pi}{4} \int_{0}^{\pi} \theta^{2} Q(d \theta)$. To prove this, we construct a jump type Markov process $\left\{X^{\varepsilon}(t)\right\}$ on $\boldsymbol{R}^{3}$ associated with (1.4) and also a diffusion process $\{X(t)\}$ on $\boldsymbol{R}^{3}$ associated with (1.6). We show that $X^{\varepsilon}(\cdot)$ converges weakly to $X(\cdot)$ as $\varepsilon$ tends to 0 which implies the convergence of the nonlinear semigroup immediately. This kind of limit theorem for Markov processes was studied well in the cases with linear infinitesimal generators by several authors (see, e.g., Kurtz [10] and Skorohod [16]). Our limit theorem is a nonlinear analogue of a part of these results.

In Section 2, we summarize known results about associated Markov processes with the Boltzmann equation and the Landau equation. Section 3 is devoted to giving estimates on the function $G(x, y, \theta ; \psi)$ and solutions of stochastic differential equations with respect to Poisson point processes. The proof of the limit theorem will be given in Section 4 . The scaling law for the measure $Q$ will be generalized when the initial distribution $f$ satisfies $\int_{\boldsymbol{R}^{\mathrm{s}}}|x|^{p} f(d x)<\infty$ with some $p>2$. Properties of the diffusion process associated with the Landau equation will be discussed in Section 5. Finally in Section 6, we refer to similar problems about the onedimensional analogous model.

The author wishes to express his thanks to Professor H. Tanaka for valuable comments.

\section{§ 2. Notations, Associated Markov Processes and Statement of the Main Result}

In this section we first fix notations to be used throughout this paper and then summarize known results about an associated Markov process 
with the Boltzmann equation. We also investigate briefly an associated diffusion process with the Landau equation. Finally, we state the main result of the present paper.

(1) Notations. (i) For $\boldsymbol{R}^{3}$-valued two random variables $X$ and $Y$ on possibly different probability spaces, we mean by $X \sim Y$ the equivalence in law. We also denote by $X \sim f$ when the probability distribution of $X$ on $\boldsymbol{R}^{3}$ is given by $f$.

(ii) Let $C \equiv C\left([0, \infty), \boldsymbol{R}^{3}\right)$ and $D \equiv D\left([0, \infty), \mathbb{R}^{3}\right)$ be the space of $\boldsymbol{R}^{3}$-valued continuous functions on $[0, \infty)$ and that of $\mathbb{R}^{3}$-valued right continuous functions on $[0, \infty)$ having left limits, respectively. We denote by $X_{t} \equiv X_{t}(w)$ the value $w(t)$ of $w \in D$ at $t \in[0, \infty)$. We set $\mathcal{B}^{C}$ and $\mathscr{B}_{t}^{C}, 0 \leqq t<\infty$, the smallest $\sigma$-fields on the space $C$ generated by $\left\{X_{s} ; 0 \leqq s<\infty\right\}$ and $\left\{X_{s} ; 0 \leqq s \leqq t\right\}$, respectively. In a similar way, $\sigma$-fields $\mathscr{B}^{D}$ and $\mathscr{B}_{t}^{D}, 0 \leqq t<\infty$, are introduced on the space $D$. In the spaces $C$ and $D$, we consider the $U$-topology, i.e., the topology given by the uniform convergence on each bounded interval of $[0, \infty)$ and the $S$-topology, i.e., the Skorohod topology (see Billingsley [3]), respectively. Then it is well-known that $C$ and $D$ are Polish spaces (i.e., completely metrizable and separable spaces) and $\mathscr{B}^{C}$ and $\mathscr{B}^{D}$ coincide with the topological Borel fields of $C$ and $D$, respectively. Occasionally we also consider the $U$-topology on the space $D$ so that we sometimes denote $D$ by $D_{S}$ or $D_{U}$ to make its topology precise. We note that the space $D_{U}$ is not separable.

(iii) Let $\mathscr{Q}_{p} \equiv \mathscr{L}_{p}\left(\boldsymbol{R}^{3}\right), 2 \leqq p<\infty$, be a family of Borel probability distributions $f$ on $\boldsymbol{R}^{3}$ which satisfy

$$
\|f\|_{p} \equiv\left\{\int_{\mathbb{R}^{3}}|x|^{p} f(d x)\right\}^{1 / p}<\infty
$$

The space $\mathscr{L}_{p}$ has a metric $\rho_{p}$ defined by

$$
\rho_{p}\left(u_{1}, u_{2}\right)=\inf \left\{\int_{\boldsymbol{R}^{\mathrm{s}}}|x-y|^{p} u(d x d y)\right\}^{1 / p}, u_{1}, u_{2} \in \mathscr{L}_{p},
$$

where the infimum is taken over all Borel probability measures $u$ on $\boldsymbol{R}^{6}$ which satisfy $u\left(B \times \boldsymbol{R}^{3}\right)=u_{1}(B)$ and $u\left(\boldsymbol{R}^{3} \times B\right)=u_{2}(B)$ for every Borel subset $B$ of $\boldsymbol{R}^{3}$. When $p=2$, we denote $\|\cdot\|_{2}$ and $\rho_{2}(\cdot, \cdot)$ simply by $\|\cdot\|$ 
and $\rho(\cdot, \cdot)$, respectively. For $f \in \mathscr{L}_{2}$, we set

$$
\begin{aligned}
& m(f)=\left\{m_{i}(f)\right\}_{i=1}^{3}=\int x f(d x) \quad(=\text { the mean vector of } f) \\
& \sigma(f)=\left\{\sigma_{i j}(f)\right\}_{i, j=1}^{3}=\left\{\int\left(x_{i}-m_{i}(f)\right)\left(x_{j}-m_{j}(f)\right) f(d x)\right\}_{i, j=1}^{3} \\
& \quad(=\text { the covariance matrix of } f), \\
& v(f)=\frac{1}{3} \sum_{i=1}^{3} \sigma_{i i}(f)
\end{aligned}
$$

and

$$
\boldsymbol{e}(f)=\rho^{2}\left(f, g_{f}\right),
$$

where $g_{f}$ is the Gaussian distribution on $\boldsymbol{R}^{3}$ with mean vector $m(f)$ and covariance matrix $v(f) \cdot \boldsymbol{I}(\boldsymbol{I}=3 \times 3$-identity matrix).

(iv) For probability distributions $P_{1}$ and $P_{2}$ on the space $\left(D, \mathcal{B}^{D}\right)$, we put

$$
\begin{gathered}
\bar{\rho}_{p, T}\left(P_{1}, P_{2}\right)=\inf \left\{\int_{D \times D} \sup _{0 \leqq t \leqq T}\left|w(t)-w^{\prime}(t)\right|^{p} P\left(d w d w^{\prime}\right)\right\}^{1 / p}, \\
p \geqq 2, T<\infty,
\end{gathered}
$$

where the infimum is taken over all probability measures $P$ on $(D \times D$, $\left.\mathscr{B}^{D} \times \mathscr{B}^{D}\right)$ which satisfy $P(B \times D)=P_{1}(B)$ and $P(D \times B)=P_{2}(B)$ for every $B \in \mathscr{B}^{D}$. When $p=2$, we denote $\bar{\rho}_{2, T}(\cdot, \cdot)$ simply by $\bar{\rho}_{T}(\cdot, \cdot)$.

(v) Let $B\left([0, \infty), \mathscr{L}_{p}\right)$ and $C\left([0, \infty), \mathscr{Q}_{p}\right), 2 \leqq p<\infty$, be the space of $\mathscr{L}_{p}$-valued Borel measurable functions $u(t)$ on $[0, \infty)$ satisfying $\sup _{0 \leqq t \leqq T}\|u(t)\|_{p}<\infty$ for every $T<\infty$ and that of $\mathscr{L}_{p}$-valued continuous functions on $[0, \infty)$, respectively. For $u(\cdot) \in B\left([0, \infty), \mathscr{L}_{2}\right)$ and $T<\infty$, we set

$$
\|u(\cdot)\|_{[0, T]}=\sup _{0 \leqq t \leqq T}\|u(t)\| .
$$

(2) Associated Markov process with the Boltzmann equation. Let $Q$ be a Borel measure on $(0, \pi)$ satisfying $\int_{0}^{\pi} \theta Q(d \theta)<\infty$. We set $S=(0, \pi) \times(0,2 \pi) \times(0,1), S_{t}=(0, t] \times S$ and $a(x, y, \theta, \phi)=x^{*}-x$. Let $N \equiv N(d s d \theta d \phi d \alpha)$ be a Poisson random measure on $(0, \infty) \times S$ with intensity measure $\widehat{N} \equiv d s Q(d \theta) d \phi d \alpha$.

For $f \in \mathscr{Q}_{2}$, we consider the following stochastic differential equation: 


$$
\left\{\begin{array}{l}
X(t)=X(0)+\int_{s_{t}} a\left(X\left(s^{-}\right), Y\left(s^{-}, \alpha\right), \theta, \phi\right) N(d s d \theta d \phi d \alpha), \\
X(0) \sim f
\end{array}\right.
$$

under the condition that $\{Y(t, \alpha) ; t \geqq 0\}$ is a right continuous $\mathbb{R}^{3}$-valued stochastic process defined on a probability space $\{(0,1), d \alpha\}$ and satisfies $Y(t, \cdot) \sim X(t)$ for each $t \geqq 0$. The following two propositions are due to Tanaka [20].

Proposition 2.1. (i) On a suitable probability space $\{\Omega, \mathscr{F}, P\}$ with an increasing family $\left\{\mathscr{F}_{t} ; t \geqq 0\right\}$ of sub- $\sigma$-fields of $\mathscr{F}$ we can construct an $\left\{\mathscr{I}_{t}\right\}$-adapted Poisson random measure $N$ (i.e., $N(B)$ is $\mathscr{F}_{t}$-measurable for each $t$ and Borel subset $B$ of $S_{t}$ ) with intensity measure $\hat{N}$ so that (2.1) has an ( $\mathscr{L}_{t}$-adapted) integrable solution $X(t)$, i.e., a solution which satisfies $E\left[\sup _{0 \leqq s \leqq t}|X(s)|\right]<\infty$ for each $t \geqq 0$.

(ii) The uniqueness in the law sense holds for integrable solutions of (2.1), that is, the probability law on $D$ of any integrable solution of (2.1) is uniquely determined by $f$.

(iii) The solution $X(t)$ has the Markov property in the sense of McKean [13].

We denote by $u(t) \equiv T_{t} f$ the probability distribution of the integrable solution $X(t)$ of (2.1).

Proposition 2.2. (i) If the initial distribution $f$ belongs to the space $\mathscr{L}_{p}(p \geqq 2)$, then $u(\cdot)$ belongs to the space $B\left([0, \infty), \mathscr{Q}_{p}\right)$.

(ii) The distribution $u(t)$ solves the equation (1.3) and $\left\{T_{t}\right.$; $t \geqq 0\}$ becomes a nonlinear semigroup on $\mathscr{P}_{2}$.

(iii) For every $t \geqq 0$, we have

$$
m(u(t))=m(f) \quad \text { and }\|u(t)\|=\|f\| .
$$

(iv) The nonlinear semigroup $\left\{T_{t}\right\}$ on $\mathscr{Q}_{2}$ is non-expansive with respect to the metric $\rho$ :

$$
\rho\left(T_{t} f_{1}, T_{t} f_{2}\right) \leqq \rho\left(f_{1}, f_{2}\right), \quad t \geqq 0, \quad f_{1}, f_{2} \in \mathscr{L}_{2} .
$$

(v) As $t \rightarrow \infty, \boldsymbol{e}\left(T_{t} f\right)$ decreases to 0 . 
The stochastic process $X(t)$ constructed as the integrable solution of (2.1) is called an associated Markov process with the Boltzmann equation (1.3). We also consider, for given $f \in \mathscr{L}_{2}$ and $u(\cdot) \in B([0$, $\infty), \mathscr{Q}_{2}$ ), the following stochastic differential equation:

$$
\left\{\begin{array}{l}
X(t)=X(0)+\int_{s_{t}} a(X(s-), Y(s-, \alpha), \theta, \phi) N(d s d \theta d \phi d \alpha) \\
Y(t, \cdot) \sim u(t), t \geqq 0 \\
X(0) \sim f
\end{array}\right.
$$

A unique integrable solution $X(t)$ of (2.2) exists in a similar sense to the equation (2.1). The probability distribution on $D$ of $X(t)$ is uniquely determined by $f, u(\cdot)$ and $Q$ so that we sometimes denote it by $P(f, u(\cdot), Q)$.

(3) Associated diffusion process with the Landau equation. Let $\left\{\mathcal{A}_{u} ; u \in \mathscr{L}_{2}\right\}$ be a family of differential operators introduced in Section 1. For $f \in \mathscr{Q}_{2}$, we consider the following martingale problem (2.3) with respect to a probability measure $P$ on $\left(C, \mathscr{B}^{C}\right)$.

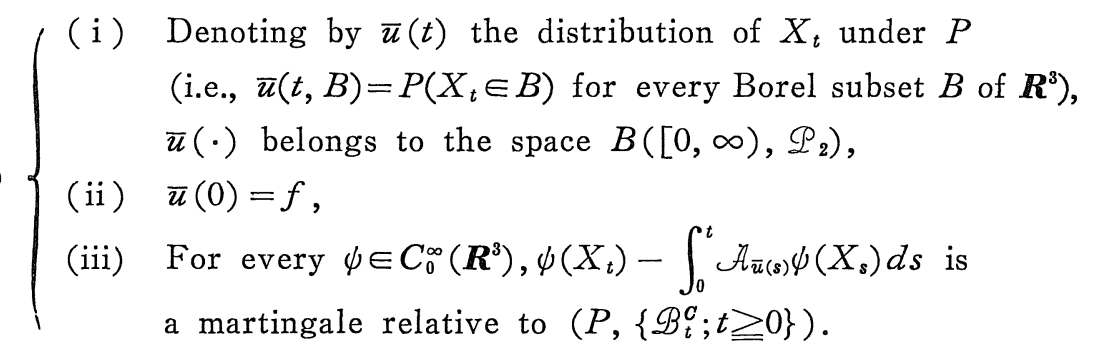

The existence of a solution to the martingale problem (2.3) can be shown by applying the result of Funaki [5]. To prove the uniqueness, let $\bar{u}(t)$ be the distribution of $X_{t}$ under some solution $P$ to the martingale problem (2.3). Then the condition (iii) proves that

(2.4) $\left\{\begin{array}{c}m(\bar{u}(t))=m(f), \quad v(\bar{u}(t))=v(f), \\ \sigma(\bar{u}(t))=v(f) \cdot \boldsymbol{I}+\{\sigma(f)-v(f) \cdot \boldsymbol{I}\} \exp (-6 \alpha t), \\ \text { for every } t \geqq 0 .\end{array}\right.$

In fact, (2.4) can be proved by showing that

$$
\frac{d}{d t} E^{P}\left[X_{t}\right]=0, \frac{d}{d t} E^{P}\left[\left|X_{t}\right|^{2}\right]=0
$$


and

$$
\begin{aligned}
& \frac{d}{d t} E^{P}\left[X_{i}(t) X_{j}(t)\right]=6 \alpha\left\{m_{i}(f) m_{j}(f)\right. \\
&\left.+\delta_{i j} v(f)-E^{P}\left[X_{i}(t) X_{j}(t)\right]\right\}, 1 \leqq i, j \leqq 3,
\end{aligned}
$$

where $E^{P}[\cdot]$ means the expectation relative to $P$ and $X_{i}(t)$ is the $i$-th component of $X_{t}$. Noting that

$$
\left\{\begin{array}{l}
\boldsymbol{a}(x, u)=\boldsymbol{a}(x-m(u))+3 \alpha v(u) \cdot \mathbf{H}-\alpha \sigma(u), \\
\boldsymbol{b}(x, u)=\boldsymbol{b}(x-m(u)),
\end{array}\right.
$$

we see that the functions $\boldsymbol{a}(t, x) \equiv \boldsymbol{a}(x, \bar{u}(t))$ and $\boldsymbol{b}(t, x) \equiv \boldsymbol{b}(x, \bar{u}(t))$ are determined only by $f$. The probability measure $P$ solves the following martingale problem:

$$
\left\{\begin{array}{l}
\text { (i) } P\left(X_{0} \in B\right)=f(B) \text { for every Borel subset } B \text { of } \mathbb{R}^{3}, \\
\text { (ii) For every } \phi \in C_{0}^{\infty}\left(\mathbb{R}^{3}\right), \psi\left(X_{t}\right)-\int_{0}^{t} \mathscr{A}_{s} \psi\left(X_{s}\right) d s \text { is a martin- } \\
\text { gale relative to }\left(P,\left\{\mathscr{B}_{t}^{c} ; t \geqq 0\right\}\right),
\end{array}\right.
$$

where

$$
\mathcal{A}_{s}=\frac{1}{2} \sum_{i, j=1}^{3} \boldsymbol{a}_{i j}(s, x) \frac{\partial^{2}}{\partial x_{i} \partial x_{j}}+\sum_{i=1}^{3} \boldsymbol{b}_{i}(s, x) \frac{\partial}{\partial x_{i}} .
$$

Since the nonnegative square root of the matrix $\boldsymbol{a}(t, x)$ is Lipschitz continuous in $x \in \mathbb{R}^{3}$ (see Theorem 5.2.3 of Stroock and Varadhan [19]), the martingale problem (2.6) is well-posed and therefore we see the uniqueness of the solution to the martingale problem (2.3). Accordingly, we obtain the following proposition combining with the results of Funaki [5].

Proposition 2.3. (i) There exists a unique probability measure $P$ on $\left(C, \mathscr{B}^{c}\right)$ which solves the martingale problem (2.3).

(ii) The solution $P$ has the Markov property in the sense of McKean and the distribution $\bar{u}(t)$ solves the equation (1.6).

(iii) If the initial distribution $f$ belongs to the space $\mathscr{L}_{p}(p \geqq 2)$, then $\bar{u}(\cdot)$ belongs to the space $C\left([0, \infty), \mathscr{P}_{p}\right)$ and we have $E^{P}\left[\sup _{0 \leqq t \leqq T}\right.$ $\left.\left|X_{t}\right|^{p}\right]<\infty$ for each $T<\infty$.

(iv) For every $t \geqq 0$, we have 


$$
m(\bar{u}(t))=m(f) \quad \text { and } \quad\|\bar{u}(t)\|=\|f\| .
$$

The stochastic process $X_{t}$ with the distribution $P$ is called an associated diffusion process with the Landau equation (1.6). We also consider, for given $f \in \mathscr{Q}_{2}$ and $u(\cdot) \in C\left([0, \infty), \mathscr{Q}_{2}\right)$, the following (2.7) called by the name of $\mathcal{A}_{u(.)}$-martingale problem.

(i) $P\left(X_{0} \in B\right)=f(B)$ for every Borel subset $B$ of $\boldsymbol{R}^{3}$. (ii) For every $\psi \in C_{0}^{\infty}\left(\boldsymbol{R}^{3}\right), \psi\left(X_{t}\right)-\int_{0}^{t} \mathcal{A}_{u(s)} \psi\left(X_{s}\right) d s$ is a martingale relative to $\left(P,\left\{\mathcal{B}_{t}^{C} ; t \geqq 0\right\}\right)$.

This martingale problem determines a unique probability measure $P$ on $\left(C, \mathscr{B}^{C}\right)$.

(4) The main result. Let $\left\{Q_{\varepsilon} ; 0<\varepsilon<1\right\}$ be a family of Borel measures on $(0, \pi)$ which satisfy the following assumption (I) or (II).

(i) $Q_{\varepsilon}((\varepsilon \pi, \pi))=0$ and $\int_{0}^{\pi} \theta Q_{\varepsilon}(d \theta)<\infty$ for $0<\varepsilon<1$.

(ii) There exists a $\operatorname{limit}_{\pi} c=\lim _{\varepsilon \downarrow 0} c_{\varepsilon}, 0<c<\infty$, where $c_{\varepsilon}=\varepsilon^{-2} \int_{0}^{\pi} \theta^{2} Q_{\varepsilon}(d \theta)$.

$$
\left\{\begin{array}{l}
\text { There exists a Borel measure } Q \text { on }(0, \pi) \text { such that } \\
\int_{0}^{\pi} \theta Q(d \theta)<\infty \text { and } Q_{\varepsilon}(B)=Q\left(\varepsilon^{-1} B \cap(0, \pi)\right) \text { holds for every } \\
\varepsilon: 0<\varepsilon<1 \text { and Borel subset } B \text { of }(0, \pi) .
\end{array}\right.
$$

If $\left\{Q_{\varepsilon}\right\}$ satisfies the assumption (II), then it fulfills also the assumption (I) with $c=\int_{0}^{\pi} \theta^{2} Q(d \theta)$. As is stated in Section 1, we consider the scaling defined by $t \rightarrow \varepsilon^{-2} t$ and $Q \rightarrow Q_{\varepsilon}$. After the transformation, the Boltzmann equation turns into the equation (1.4) and an associated Markov process $X^{\varepsilon}(t)$ is given by the solution of the following stochastic differential equation:

$$
\left\{\begin{array}{l}
X^{\varepsilon}(t)=X(0)+\int_{s_{t}} a\left(X^{\varepsilon}(s-), Y^{\varepsilon}(s-, \alpha), \theta, \phi\right) N_{\varepsilon}(d s d \theta d \phi d \alpha) \\
X^{\varepsilon}(t) \sim Y^{\varepsilon}(t, \cdot)
\end{array}\right.
$$

where $N_{\varepsilon}$ is a Poisson random measure on $(0, \infty) \times S$ with intensity measure $\widehat{N}_{\varepsilon}=\varepsilon^{-2} d s Q_{\varepsilon}(d \theta) d \phi d \alpha$. We denote by $P_{f}^{\varepsilon}$ the distribution on the space $D$ of the integrable solution $X^{\varepsilon}(\cdot)$ of $(2.8)$ with an initial 
distribution $f \in \mathscr{P}_{2}$.

Remark. The conditions $\int_{0}^{\pi} \theta Q_{\varepsilon}(d \theta)<\infty$ in (I) and $\int_{0}^{\pi} \theta Q(d \theta)<\infty$ in (II) are assumed only to use the result of Tanaka [20] which guarantees the solvability of the equation (2.8). But his unpublished result shows that these assumptions are unnecessary (private communication). See also Theorem 2 of Tanaka [22].

We also denote by $P_{f}$ the unique solution $P$ to the martingale problem (2.3) with $\alpha=c \pi / 4$ extending it on the space $\left(D, \mathscr{B}^{D}\right)$. Our main result is formulated as follows.

Theorem 2.1. (i) For each $f \in \mathscr{L}_{p}(p>2)$, under the assumption $(I)$ on $\left\{Q_{\varepsilon}\right\}$, the probability measure $P_{f}^{\varepsilon}$ converges weakly to $P_{f}$ on the space $D_{S}$ as $\varepsilon$ tends to 0 .

(ii) For each $f \in \mathscr{Q}_{2}$, under the assumption $(I I)$ on $\left\{Q_{\varepsilon}\right\}$, the probability measure $P_{f}^{\varepsilon}$ converges weakly to $P_{f}$ on the space $D_{S}$ as $\varepsilon$ tends to 0 .

\section{§3. Estimates on the Function $G(x, y, \theta ; \psi)$ and the Solution of $(2.2)$}

We prepare some estimates on the function $G$ and the solution of the stochastic differential equation (2.2) to give a proof of Theorem 2.1 in the next section.

(1) Estimates on the function $G(x, y, \theta ; \psi)$. The function $G$ was introduced in Section 1 by

$$
\begin{aligned}
& G(x, y, \theta ; \phi)=\int_{0}^{2 \pi}\left\{\psi\left(x^{*}\right)-\psi(x)\right\} d \phi, \\
&(x, y, \theta, \psi) \in \mathbb{R}^{3} \times \mathbb{R}^{3} \times(0, \pi) \times C_{0}^{\infty}\left(\mathbb{R}^{3}\right) .
\end{aligned}
$$

We sometimes denote $x^{*}$ and $e(\phi)$ by $x^{*}(x, y, \theta, \phi)$ and $e(\phi ; x, y)$ to make precise their dependences on $(x, y, \theta, \phi) \in \mathbb{R}^{3} \times \mathbb{R}^{3} \times(0, \pi) \times(0,2 \pi)$ and $(x, y) \in \mathbb{R}^{3} \times \boldsymbol{R}^{3}$, respectively. We extend $x^{*}(x, y, \theta, \phi)$ and $e(\phi ; x, y)$ 
as functions of $\phi$ to periodic functions on $\boldsymbol{R}^{1}$ with period $2 \pi$. Assuming that, for each $x \in \boldsymbol{R}^{3}, e(\phi ; x, x)$ is also given in such a way that $\frac{d e}{d \phi}=z \times e$ for some $z \in \boldsymbol{R}^{3} ;|z|=1$, we define $\phi_{0}\left(x, y ; x^{\prime}, y^{\prime}\right) \in[0,2 \pi)$, $\left(x, y, x^{\prime}, y^{\prime}\right) \in\left(\boldsymbol{R}^{3}\right)^{4}$, so that the following relation holds.

$$
\begin{gathered}
e(\bar{\phi} ; x, y)=e\left(\bar{\phi}+\phi_{0}\left(x, y ; x^{\prime}, y^{\prime}\right) ; x^{\prime}, y^{\prime}\right) \\
\text { for some } \bar{\phi} \in[0, \pi) .
\end{gathered}
$$

We note that the relation (3.1) determines $\phi_{0}\left(x, y ; x^{\prime}, y^{\prime}\right)$ uniquely.

Lemma 3. 1. For every $x, y, x^{\prime}, y^{\prime} \in \boldsymbol{R}^{3}, \theta \in(0, \pi)$ and $\phi \in(0,2 \pi)$, we have

$$
\begin{aligned}
\mid a(x, y, \theta, \phi) & -a\left(x^{\prime}, y^{\prime}, \theta, \phi+\phi_{0}\left(x, y ; x^{\prime}, y^{\prime}\right)\right) \mid \\
& \leqq \\
& \left\{x-x^{\prime}|+| y-y^{\prime} \mid\right\} \theta .
\end{aligned}
$$

Proof. Since we see that

$$
\begin{aligned}
& \max _{0 \leqq \phi \leqq 2 \pi}|e(\phi ; x, y)| x-y\left|-e\left(\phi+\phi_{0}\left(x, y ; x^{\prime}, y^{\prime}\right) ; x^{\prime}, y^{\prime}\right)\right| x^{\prime}-y^{\prime}|| \\
& \quad=\left|e\left(\bar{\phi}+\frac{\pi}{2} ; x, y\right)\right| x-y\left|-e\left(\bar{\phi}+\phi_{0}\left(x, y ; x^{\prime}, y^{\prime}\right)+\frac{\pi}{2} ; x^{\prime}, y^{\prime}\right)\right| x^{\prime}-y^{\prime}|| \\
& \quad=\left|(x-y)-\left(x^{\prime}-y^{\prime}\right)\right|,
\end{aligned}
$$

with $\bar{\phi}$ appeared in (3.1), the relation

$$
a(x, y, \theta, \phi)=(y-x) \sin ^{2} \frac{\theta}{2}+\frac{1}{2} e(\phi ; x, y)|x-y| \sin \theta
$$

proves the desired inequality.

Q.E.D.

The function $G(x, y, \theta ; \psi)$ has the following estimates.

Lemma 3.2. For each $\psi \in C_{0}^{\infty}\left(\boldsymbol{R}^{3}\right)$, there exists a positive constant $K \equiv K(\psi)$ such that

(i) $|G(x, y, \theta ; \psi)| \leqq K \theta^{2}\left\{1+|x|^{2}+\mid y_{1}^{\prime 2}\right\}$,

(ii) $\left|G(x, y, \theta ; \psi)-G\left(x^{\prime}, y, \theta ; \psi\right)\right| \leqq K \theta^{2}\left\{1+|x|^{2}+\left|x^{\prime}\right|^{2}+|y|^{2}\right\}\left|x-x^{\prime}\right|$.

We also have that

(iii) $G(x, y, \theta ; \psi)=0$ if $|x| \geqq 3 M(\psi),|y| \leqq M(\psi) / 3 \theta$ and $\theta \in(0$, 
$\pi / 2]$, where $M(\psi)=\inf \{M>0 ; \psi(x)=0$ for $|x| \geqq M\}$.

Proof. (i) For each $x, y, \theta, \phi$ and $\psi$, there exists $x^{0} \in \mathbb{R}^{3}$ such that

$$
\begin{aligned}
\psi\left(x^{*}\right)-\psi(x)= & \left\langle\nabla \psi(x), x^{*}-x\right\rangle \\
& +\frac{1}{2} \sum_{i, j=1}^{3} \frac{\partial^{2} \psi}{\partial x_{i} \partial x_{j}}\left(x^{0}\right)\left(x_{i}^{*}-x_{i}\right)\left(x_{j}^{*}-x_{j}\right),
\end{aligned}
$$

where $\nabla \psi(x)=\left(\frac{\partial \psi}{\partial x_{1}}, \frac{\partial \psi}{\partial x_{2}}, \frac{\partial \psi}{\partial x_{3}}\right)$ and $\langle\cdot, \cdot\rangle$ means the inner product of $\boldsymbol{R}^{3}$. Noting that

$$
\int_{0}^{2 \pi} e(\phi) d \phi=0 \text { and }\left|x^{*}-x\right|=|x-y| \sin \frac{\theta}{2},
$$

we get

$$
\int_{0}^{2 \pi}\left(x^{*}-x\right) d \phi=2 \pi(y-x) \sin ^{2} \frac{\theta}{2}
$$

and

$$
\int_{0}^{2 \pi}\left|\left(x_{i}^{*}-x_{i}\right)\left(x_{j}^{*}-x_{j}\right)\right| d \phi \leqq \pi \theta^{2}|x-y|^{2} / 2,1 \leqq i, j \leqq 3,
$$

respectively. Therefore, we obtain

$$
\begin{aligned}
& |G(x, y, \theta ; \psi)| \\
& \quad \leqq \frac{\pi}{2} \theta^{2}\left\{|y-x| \sup _{x}|\nabla \psi(x)|+\frac{9}{2}|y-x|^{2} \sup _{i, j, x}\left|\frac{\partial^{2} \psi}{\partial x_{i} \partial x_{j}}(x)\right|\right\},
\end{aligned}
$$

which implies the estimate (i).

(ii) For given $x, x^{\prime}, y, \theta$ and $\phi$, we denote $x^{*}(x, y, \theta, \phi)$ and $x^{*}$ $\left(x^{\prime}, y, \theta, \phi+\phi_{0}\left(x, y ; x^{\prime}, y\right)\right)$ simply by $x^{*}$ and $\left(x^{\prime}\right)^{*}$, respectively. Then we get

(3.4) $G(x, y, \theta ; \psi)-G\left(x^{\prime}, y, \theta ; \psi\right)$

$$
\begin{aligned}
& =\int_{0}^{2 \pi}\left\{\psi\left(x^{*}\right)-\psi\left(\left(x^{\prime}\right)^{*}\right)-\psi(x)+\psi\left(x^{\prime}\right)\right\} d \phi \\
& =\int_{0}^{2 \pi} d \phi \int_{0}^{1}\left\langle\nabla \psi\left(z^{*}(\gamma, \phi)\right), x^{*}-\left(x^{\prime}\right)^{*}\right\rangle d \gamma \\
& \quad-\int_{0}^{2 \pi} d \phi \int_{0}^{1}\left\langle\nabla \psi(z(\gamma)), x-x^{\prime}\right\rangle d \gamma
\end{aligned}
$$




$$
\begin{aligned}
= & \int_{0}^{2 \pi} d \phi \int_{0}^{1}\left\langle\nabla \psi\left(z^{*}(\gamma, \phi)\right)-\nabla \psi(z(\gamma)), x^{*}-\left(x^{\prime}\right)^{*}-x+x^{\prime}\right\rangle d \gamma \\
& +\int_{0}^{2 \pi} d \phi \int_{0}^{1}\left\langle\nabla \psi\left(z^{*}(\gamma, \phi)\right)-\nabla \psi(z(\gamma)), x-x^{\prime}\right\rangle d \gamma \\
& +\int_{0}^{2 \pi} d \phi \int_{0}^{1}\left\langle\nabla \psi(z(\gamma)), x^{*}-\left(x^{\prime}\right)^{*}-x+x^{\prime}\right\rangle d \gamma \\
\equiv & I_{1}+I_{2}+I_{3},
\end{aligned}
$$

where

$$
z^{*}(\gamma, \phi)=\gamma x^{*}+(1-\gamma)\left(x^{\prime}\right)^{*}
$$

and

$$
z(\gamma)=\gamma x+(1-\gamma) x^{\prime}, 0 \leqq \gamma \leqq 1 .
$$

To give a bound on $I_{1}$, we derive

$$
\begin{aligned}
& \left|z^{*}(\gamma, \phi)-z(\gamma)\right| \\
& \quad=\left|\gamma\left\{x^{*}-x\right\}+(1-\gamma)\left\{\left(x^{\prime}\right)^{*}-x^{\prime}\right\}\right| \\
& \quad \leqq\left\{\gamma|x-y|+(1-\gamma)\left|x^{\prime}-y\right|\right\} \sin \frac{\theta}{2} \\
& \quad \leqq \theta\left\{|x|+\left|x^{\prime}\right|+|y|\right\},
\end{aligned}
$$

and, by Lemma 3.1,

$$
\begin{aligned}
\mid x^{*}- & \left(x^{\prime}\right)^{*}-x+x^{\prime} \mid \\
& =\left|a(x, y, \theta, \phi)-a\left(x^{\prime}, y, \theta, \phi+\phi_{0}\left(x, y ; x^{\prime}, y\right)\right)\right| \\
& \leqq \theta\left|x-x^{\prime}\right| .
\end{aligned}
$$

Two estimates (3.5) and (3.6) show that

$$
\left|I_{1}\right| \leqq 6 \pi \theta^{2}\left\{|x|+\left|x^{\prime}\right|+|y|\right\}\left|x-x^{\prime}\right| \sup _{i, j, x}\left|\frac{\partial^{2} \psi}{\partial x_{i} \partial x_{j}}(x)\right| .
$$

Noting that

$$
\int_{0}^{2 \pi}\left(z^{*}(\gamma, \phi)-z(\gamma)\right) d \phi=2 \pi\{y-z(\gamma)\} \sin ^{2} \frac{\theta}{2}
$$

and

$$
\int_{0}^{2 \pi}\left|\left(z_{i}^{*}(\gamma, \phi)-z_{i}(\gamma)\right)\left(z_{j}^{*}(\gamma, \phi)-z_{j}(\gamma)\right)\right| d \phi
$$




$$
\leqq 2 \pi \theta^{2}\left\{|x|+\left|x^{\prime}\right|+|y|\right\}^{2}
$$

the second term $I_{2}$ can be bounded in a similar manner to show the estimate (i) and accordingly we get

$$
\begin{aligned}
\left|I_{2}\right| \leqq & 2 \pi \theta^{2}\left\{\left(\left|x_{i}\right|+\left|x^{\prime}\right|+|y|\right) \sup _{i, j, x}\left|\frac{\partial^{2} \psi}{\partial x_{i} \partial x_{j}}(x)\right|\right. \\
& \left.+9\left(|x|+\left|x^{\prime}\right|+|y|\right)^{2} \sup _{i, j, x, x}\left|\frac{\partial^{3} \psi}{\partial x_{i} \partial x_{j} \partial x_{k}}(x)\right|\right\}\left|x-x^{\prime}\right| .
\end{aligned}
$$

As for the third term $I_{3}$, since

$$
I_{3}=2 \pi \sin ^{2} \frac{\theta}{2}\left\{\psi\left(x^{\prime}\right)-\psi(x)\right\},
$$

we have

$$
\left|I_{3}\right| \leqq \frac{\pi}{2} \theta^{2}\left|x-x^{\prime}\right| \sup _{x}|\nabla \psi(x)|
$$

Combining the three estimates (3.7)-(3.9) with (3.4), we obtain the desired estimate (ii).

(iii) Noting the relation $\left|x^{*}-x\right|=\left|x^{*}-y\right| \tan \frac{\theta}{2} \leqq \theta\left|x^{*}-y\right|(\theta \in(0$, $\pi / 2]$ ), we can easily show that the conditions for $x$ and $y$ in the statement (iii) implies that $\left|x^{*}\right| \geqq M(\psi)$ which proves that $G(x, y, \theta ; \psi)=0$.

Q.E.D.

(2) Estimates on the solution of (2.2). Here, we fix a positive number $p \geqq 2$. For given $f \in \mathscr{L}_{p}$ and $u(\cdot) \in B\left([0, \infty), \mathscr{L}_{2}\right)$, we consider the stochastic differential equation (2.2) and derive estimates on the integrable solution $X(t)$ defined on a complete probability space $(\Omega, \mathscr{F}$, $\left.P ;\left\{\mathscr{I}_{t} ; t \geqq 0\right\}\right)$. We set $c=\int_{0}^{\pi} \theta^{2} Q(d \theta)$ and denote by $\widetilde{u}(t)$ the probability distribution of $X(t)$ on $\mathbb{R}^{3}$.

Lemma 3.3. (i) For each $T, U$ and $c_{0}>0$, there exists a positive constant $K_{1} \equiv K_{1}\left(T, U, c_{0}, p\right)$ such that an inequality

$$
E\left[\sup _{0 \leqq t \leqq T}|X(t)|^{p}\right] \leqq K_{1}\left\{1+\|f\|_{p}^{p}\right\}
$$

holds whenever $c \in\left(0, c_{0}\right]$ and $\|u(\cdot)\|_{[0, T]} \leqq U$. 
(ii) For every $s \geqq 0$, we have

$$
\lim _{t \rightarrow s} E\left[|X(t)-X(s)|^{p}\right]=0 .
$$

Especially, the function $\widetilde{u}(\cdot)$ belongs to the space $C\left([0, \infty), \mathscr{L}_{p}\right)$.

(iii) For each $T$ and $c_{0}>0$, there exists a positive constant $K_{2}$ $\equiv K_{2}\left(T, c_{0}\right)$ such that

$$
\begin{gathered}
E\left[\left|X\left(t \wedge \zeta_{n}\right)-X\left(s \wedge \zeta_{n}\right)\right|^{2} \mid \mathscr{I}_{s}\right] \leqq K_{2}\left\{n^{2}+\|u(\cdot)\|_{[0, T]}^{2}\right\}(t-s), \\
0 \leqq s \leqq t \leqq T, n=1,2, \cdots, P \text {-a.s., }
\end{gathered}
$$

holds whenever $c \in\left(0, c_{0}\right]$, where $\zeta_{n}(\omega)=\inf \{t>0 ;|X(t ; \omega)|>n\}, \omega \in \Omega$, and $t \wedge \zeta=\min (t, \zeta)$.

Proof. (i) We set $M(t)=\int_{s_{t}} a(s, \theta, \phi, \alpha)\{N-\widehat{N}\}(d s d \theta d \phi d \alpha)$, $A(t)=\int_{s_{t}} a(s, \theta, \phi, \alpha) \hat{N}(d s d \theta d \phi d \alpha)$, where $a(s, \theta, \phi, \alpha) \equiv a(s, \theta, \phi, \alpha ; \omega)$ $=a(X(s-), Y(s-, \alpha), \theta, \phi)$. Then $M(t)$ is a 3-dimensional martingale relative to $\left(P,\left\{\mathscr{F}_{t} ; t \geqq 0\right\}\right)$ and therefore Burkholder-Davis-Gundy's inequality (see Dellacherie et Meyer [4] or Kazamaki [9]) yields an bound

$$
E\left[\sup _{0 \leqq s \leqq t \wedge \zeta_{n}}|M(s)|^{p}\right] \leqq C_{1} E\left[\left|[M]\left(t \wedge \zeta_{n}\right)\right|^{p / 2}\right]
$$

with some positive constant $C_{1} \equiv C_{1}(p)$ where $[M](t) \equiv\left\{[M]_{i}(t)\right\}_{i=1}^{3}$ is defined by

$$
[M]_{i}(t)=\int_{S_{t}} a_{i}^{2}(s, \theta, \phi, \alpha) N(d s d \theta d \phi d \alpha), 1 \leqq i \leqq 3,
$$

and $a_{i}$ is the $i$-th component of the vector $a$. Since the relation $|a(x, y, \theta, \phi)|=|x-y| \sin \frac{\theta}{2}$ implies that

$$
|[M](t)| \leqq \int_{s_{t}}\left\{\left|X\left(s^{-}\right)\right|^{2}+|Y(s-, \alpha)|^{2}\right\} \theta^{2} N(d s d \theta d \phi d \alpha),
$$

to bound the right hand side of (3.10), we set

$$
I_{1}(t)=\left\{\int_{s_{t}}\left|X\left(s^{-}\right)\right|^{2} \theta^{2} N(d s d \theta d \phi d \alpha)\right\}^{p / 2} \equiv\left\{Z_{1}(t)\right\}^{p / 2}
$$

and

$$
I_{2}(t)=\left\{\int_{s_{t}}|Y(s-, \alpha)|^{2} \theta^{2} N(d s d \theta d \phi d \alpha)\right\}^{p / 2} \equiv\left\{Z_{2}(t)\right\}^{p / 2}
$$


Itô's formula (see, e.g., Ikeda and Watanabe [6]) yields that

$$
\begin{aligned}
I_{1}(t) & =\int_{s_{t}}\left[\left\{Z_{1}\left(s^{-}\right)+\left|X\left(s^{-}\right)\right|^{2} \theta^{2}\right\}^{p / 2}-Z_{1}\left(s^{-}\right)^{p / 2}\right] N(d s d \theta d \phi d \alpha) \\
& \leqq p \int_{s_{t}}\left|X\left(s^{-}\right)\right|^{2} \theta^{2}\left\{Z_{1}\left(s^{-}\right)+\left|X\left(s^{-}\right)\right|^{2} \theta^{2}\right\}^{(p / 2)-1} N(d s d \theta d \phi d \alpha) \\
& \leqq p 2^{p} \int_{s_{t}}\left\{\left|X\left(s^{-}\right)\right|^{2} \theta^{2} Z_{1}\left(s^{-}\right)^{(p / 2)-1}+\left|X\left(s^{-}\right)\right|^{p} \theta^{p}\right\} N(d s d \theta d \phi d \alpha) \\
& \leqq C_{2} \int_{s_{t}}\left\{I_{1}\left(s^{-}\right) \theta^{2}+\left|X\left(s^{-}\right)\right|^{p} \theta^{2}\right\} N(d s d \theta d \phi d \alpha)
\end{aligned}
$$

where $C_{2}$ is a positive constant which depends only on $p$. Hence we get

$$
\begin{aligned}
E\left[I_{1}\left(t \wedge \zeta_{n}\right)\right] & \leqq C_{2} \int_{S_{t}} \theta^{2} E\left[I_{1}\left(s \wedge \zeta_{n^{-}}\right)+\left|X\left(s \wedge \zeta_{n^{-}}\right)\right|^{p}\right] \hat{N}(d s d \theta d \phi d \alpha) \\
& \leqq 2 \pi c C_{2} \int_{0}^{t}\left\{E\left[I_{1}\left(s \wedge \zeta_{n}\right)\right]+E\left[\left|X\left(s \wedge \zeta_{n^{-}}\right)\right|^{p}\right]\right\} d s
\end{aligned}
$$

which implies, by Gronwall's lemma,

$$
E\left[I_{1}\left(t \wedge \zeta_{n}\right)\right] \leqq 2 \pi c C_{2} e^{2 \pi c C_{2} t} \int_{0}^{t} E\left[\left|X\left(s \wedge \zeta_{n^{-}}\right)\right|^{p}\right] d s
$$

To get an estimate on $E\left[I_{2}\left(t \wedge \zeta_{n}\right)\right]$, we note the following fact, that is, if $X$ is a Poisson random variable with mean $\mu>0$, then, for each $\mu_{0}>0$, there exists a positive constant $C_{3} \equiv C_{3}\left(\mu_{0}, p\right)$ such that $E\left[X^{p}\right] \leqq C_{3} \mu$ holds whenever $\mu \in\left(0, \mu_{0}\right)$. This is shown by noting that $E\left[X^{p}\right]=\mu E[(X$ $\left.+1)^{p-1}\right]$ holds for every $p \geqq 1$. Since $Z_{2}(t)$ is Poisson distributed with mean $2 \pi c \int_{0}^{t}\|u(s)\|^{2} d s$, we get, for each $c_{0}$ and $U>0$,

$$
\begin{aligned}
& E\left[I_{2}\left(t \wedge \zeta_{n}\right)\right] \leqq E\left[I_{2}(t)\right] \\
& \quad \leqq C_{3}\left(2 \pi c_{0} T U^{2}, p / 2\right) 2 \pi c t U^{2}, t \leqq T,
\end{aligned}
$$

whenever $c \in\left(0, c_{0}\right]$ and $\|u(\cdot)\|_{[0, T]} \leqq U$. Combining the estimates (3.11) and (3.12) with (3.10), we see that, for each $T, U$ and $c_{0}>0$, there exists a positive constant $C_{4}$ such that

$$
\begin{gathered}
E\left[\sup _{0 \leqq s \leqq t \wedge \zeta_{n}}|M(s)|^{p}\right] \leqq C_{4}\left\{\int_{0}^{t} E\left[\left|X\left(s \wedge \zeta_{n^{-}}\right)\right|^{p}\right] d s+t\right\}, \\
0 \leqq t \leqq T, n=1,2, \cdots,
\end{gathered}
$$

holds whenever $c \in\left(0, c_{0}\right]$ and $\|u(\cdot)\|_{[0, T]} \leqq U$. While, noting that the 
relation $\int_{0}^{2 \pi} e(\phi) d \phi=0$ implies that

$$
A(t)=\int_{s_{t}}\left\{Y\left(s^{-}, \alpha\right)-X\left(s^{-}\right)\right\} \sin ^{2} \frac{\theta}{2} \hat{N}(d s d \theta d \phi d \alpha),
$$

we can show a similar bound on $A(t)$, that is, for each $T, U$ and $c_{0}>0$, there exists a positive constant $C_{5}$ such that

$$
\begin{gathered}
E\left[\sup _{0 \leqq s \leqq t \wedge \zeta_{n}}|A(s)|^{p}\right] \leqq C_{5}\left\{\int_{0}^{t} E\left[\left|X\left(s \wedge \zeta_{n^{-}}\right)\right|^{p}\right] d s+1\right\}, \\
0 \leqq t \leqq T, n=1,2, \cdots,
\end{gathered}
$$

holds whenever $c \in\left(0, c_{0}\right]$ and $\|u(\cdot)\|_{[0, T]} \leqq U$. Since $X(t)=X(0)+M(t)$ $+A(t)$, two estimates (3.13) and (3.14) yield an estimate on $X(t)$ with some positive constant $C_{6} \equiv C_{6}\left(T, U, c_{0}, p\right)$ :

$$
\begin{gathered}
E\left[\sup _{0 \leqq s \leqq t \wedge \zeta_{n}}|X(s)|^{p}\right] \leqq C_{6}\left\{1+\|f\|_{p}^{p}+\int_{0}^{t} E\left[\left|X\left(s \wedge \zeta_{n^{-}}\right)\right|^{p}\right] d s\right\} \\
\quad \leqq C_{6}\left\{1+\|f\|_{p}^{p}+\int_{0}^{t} E\left[\sup _{0 \leqq r \leqq s \wedge \zeta_{n}}|X(r)|^{p}\right] d s\right\} .
\end{gathered}
$$

Therefore Gronwall's lemma shows that

$$
\begin{aligned}
& E\left[\sup _{0 \leqq s \leqq T \wedge \zeta_{n}}|X(s)|^{p}\right] \leqq C_{6}\left\{1+\|f\|_{p}^{p}\right\} e^{C_{6} T}, n=1,2, \cdots, \\
& \text { for } c \in\left(0, c_{0}\right] \text { and }\|u(\cdot)\|_{[0, T] \leqq U .}
\end{aligned}
$$

Tending $n$ to infinity, we get the estimate (i) by Fatou's lemma.

(ii) Burkholder-Davis-Gundy's inequality shows that

$$
\begin{aligned}
& E\left[|M(t)-M(s)|^{p}\right] \\
& \quad \leqq C_{4}\left\{\int_{s}^{t} E\left[|X(r-)|^{p}\right] d r+(t-s)\right\} \rightarrow 0 \text { as }|t-s| \rightarrow 0 \quad(0 \leqq s \leqq t \leqq T)
\end{aligned}
$$

with the same constant $C_{4}$ in $(3.13)$. We can also show that

$$
\begin{aligned}
& E\left[|A(t)-A(s)|^{p}\right] \\
& \qquad C_{7} c^{p}|t-s|^{p-1} \int_{s}^{t}\left\{E\left[|X(r)|^{p}\right]+\|u(r)\|^{p}\right\} d r \\
& \quad \rightarrow 0 \text { as }|t-s| \rightarrow 0 \quad(0 \leqq s \leqq t \leqq T)
\end{aligned}
$$

with some positive constant $C_{7} \equiv C_{7}(p)$. Hence we have

$$
\lim _{|t-s| \rightarrow 0} E\left[|X(t)-X(s)|^{p}\right]=0 .
$$


Noting that $\rho_{p}(\widetilde{u}(t), \widetilde{u}(s)) \leqq E\left[|X(t)-X(s)|^{p}\right]^{1 / p}$, we see that $\widetilde{u}(\cdot)$ is

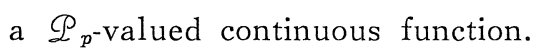

(iii) We observe that

$$
\begin{aligned}
& E\left[\left|X\left(t \wedge \zeta_{n}\right)-X\left(s \wedge \zeta_{n}\right)\right|^{2} \mid \mathscr{F}_{s}\right] \\
& \leqq 2\left\{E\left[\left|M\left(t \wedge \zeta_{n}\right)-M\left(s \wedge \zeta_{n}\right)\right|^{2} \mid \mathscr{E}_{s}\right]\right. \\
&\left.+E\left[\left|A\left(t \wedge \zeta_{n}\right)-A\left(s \wedge \zeta_{n}\right)\right|^{2} \mid \mathscr{E}_{s}\right]\right\} \\
& \equiv 2\left\{I_{1}^{\prime}+I_{2}^{\prime}\right\} .
\end{aligned}
$$

The first term has the following bound:

$$
\begin{aligned}
& I_{1}^{\prime}=E\left[\int_{S_{t \wedge \zeta_{n}}-S_{s \wedge \zeta_{n}}}|a(r, \theta, \phi, \alpha)|^{2} \hat{N}(d r d \theta d \phi d \alpha) \mid \mathscr{I}_{s}\right] \\
& \quad \leqq c \pi E\left[\int_{s \wedge \zeta_{n}}^{t \wedge \zeta_{n}}\left\{|X(r-)|^{2}+\|u(r)\|^{2}\right\} d r \mid \mathscr{I}_{s}\right] \\
& \quad \leqq c \pi\left\{n^{2}+\|u(\cdot)\|_{[0, T]}^{2}\right\}(t-s), 0 \leqq s \leqq t \leqq T \quad(P \text {-a.s. }) .
\end{aligned}
$$

While the second term is bounded as follows.

$$
\begin{aligned}
I_{2}^{\prime} & =E\left[\left|\int_{S_{t \wedge \zeta_{n}}-S_{s \wedge \zeta_{n}}} a(r, \theta, \phi, \alpha) \hat{N}(d r d \theta d \phi d \alpha)\right|^{2} \mid \mathscr{E}_{s}\right] \\
& =E\left[\left|\int_{S_{t \wedge \zeta_{n}}-S_{s \wedge \zeta_{n}}}\{Y(r-, \alpha)-X(r-)\} \sin ^{2} \frac{\theta}{2} \widehat{N}(d r d \theta d \phi d \alpha)\right|^{2} \mid \mathscr{E}_{s}\right] \\
& \leqq\left\{c \pi\left(n+\|u(\cdot)\|_{[0, T]}\right)(t-s) / 2\right\}^{2}, 0 \leqq s \leqq t \leqq T \quad(P \text {-a. s. }) .
\end{aligned}
$$

Hence we get the estimate (iii) combining those on $I_{1}^{\prime}$ and $I_{2}^{\prime}$ with (3.16).

Q.E.D.

The next task is to give an estimate on $P(f, u(\cdot), Q)$ with respect to $u(\cdot)$ where $P(f, u(\cdot), Q)$ is the probability distribution on $D$ introduced by solving the equation (2.2) (see Section 2). Let $\widetilde{C}([0, \infty)$, $\left.\mathscr{L}_{2}\right)$ be the space of all $u(\cdot) \in C\left([0, \infty), \mathscr{L}_{2}\right)$, for each of which there exists a stochastic process $Y(t, \alpha)$ defined on $\{(0,1), d \alpha\}$ satisfying the following condition.

$$
\left\{\begin{array}{l}
\text { (i) } Y(t, \cdot) \sim u(t) \text { for every } t \in[0, \infty), \\
\text { (ii) } \lim _{t \rightarrow s} \int_{0}^{1}|Y(t, \alpha)-Y(s, \alpha)|^{2} d \alpha=0 \text { for every } s \in[0, \infty) .
\end{array}\right.
$$


Lemma 3.4. For each $T$ and $c_{0}>0$, there exists a positive constant $K_{3} \equiv K_{3}\left(T, c_{0}\right)$ such that

$$
\bar{\rho}_{t}^{2}\left(P, P^{\prime}\right) \leqq K_{3} \int_{0}^{t} \rho^{2}\left(u(s), u^{\prime}(s)\right) d s, t \leqq T,
$$

holds for all $u(\cdot)$ and $u^{\prime}(\cdot) \in \widetilde{C}\left([0, \infty), \mathscr{L}_{2}\right)$ whenever $c \in\left(0, c_{0}\right]$, where $P=P(f, u(\cdot), Q)$ and $P^{\prime}=P\left(f, u^{\prime}(\cdot), Q\right)$.

Proof. We fix $f \in \mathscr{Q}_{2}$. Let $\Delta$ be a partition of $[0, T] ; 0=t_{0}<t_{1}$ $<\cdots<t_{n}=T$ and put $\Delta(s)=t_{k}$ for $t_{k}<s \leqq t_{k+1}(0 \leqq k<n),|\Delta|=\max \left\{t_{k+1}\right.$ $\left.-t_{k} ; 0 \leqq k<n\right\}$. For $u(\cdot) \in \widetilde{C}\left([0, \infty), \mathscr{Q}_{2}\right)$, taking a stochastic process $Y(t, \alpha)$ which satisfies the condition $(3.17)$, we define $X_{\Delta}(t)$ by

$$
\left\{\begin{array}{l}
X_{\Delta}(t)=X(0)+\int_{s_{t}} a_{\Delta}(s, \theta, \phi, \alpha) N(d s d \theta d \phi d \alpha) \\
a_{\Delta}(s, \theta, \phi, \alpha)=a\left(X_{\Delta}(\Delta(s)), Y(\Delta(s), \alpha), \theta, \phi\right)
\end{array}\right.
$$

where $X(0)$ is an $\mathscr{E}_{0}$-measurable f-distributed random variable. The probability distribution of $X_{\Delta}(\cdot)$ on the space $D$ is determined uniquely by $u(\cdot)$ and $\Delta$ so that we denote it by $P(u(\cdot), \Delta)$. First we show that

$$
\lim _{|\Delta| \rightarrow 0} \bar{\rho}_{T}(P(u(\cdot), \Delta), P(f, u(\cdot), Q))=0 .
$$

To this end, we note that the probability distribution of $\bar{X}_{\Delta}(\cdot)$ defined by

$$
\left\{\begin{array}{l}
\bar{X}_{\Delta}(t)=X(0)+\int_{S_{t}} \bar{a}_{\Delta}(s, \theta, \phi, \alpha) N(d s d \theta d \phi d \alpha), \\
\bar{a}_{\Delta}(s, \theta, \phi, \alpha) \\
\quad=a\left(\bar{X}_{\Delta}(\Delta(s)), Y(\Delta(s), \alpha), \theta, \phi+\phi_{0}\left(X(s-), Y(s-, \alpha), \bar{X}_{\Delta}(\Delta(s)), Y(\Delta(s), \alpha)\right)\right),
\end{array}\right.
$$

is also given by $P(u(\cdot), \Delta)$, where $X(t)$ is the solution of (2.2) with $X(0)$ and $Y(t, \alpha)$ given as above. We set

$$
\begin{aligned}
& a(s, \theta, \phi, \alpha)=a(X(s-), Y(s-, \alpha), \theta, \phi), \\
& M_{\Delta}(t)=\int_{s_{t}}\left\{\bar{a}_{\Delta}(s, \theta, \phi, \alpha)-a(s, \theta, \phi, \alpha)\right\}(N-\widehat{N})(d s d \theta d \phi d \alpha), \\
& A_{\Delta}(t)=\int_{s_{t}}\left\{\bar{a}_{\Delta}(s, \theta, \phi, \alpha)-a(s, \theta, \phi, \alpha)\right\} \hat{N}(d s d \theta d \phi d \alpha)
\end{aligned}
$$

and

$$
I_{1, \Delta}(t)=\int_{0}^{1}|Y(\Delta(t), \alpha)-Y(t, \alpha)|^{2} d \alpha .
$$


Then, by Doob's inequality and Lemma 3.1, we get

$$
\begin{aligned}
& E\left[\sup _{0 \leqq s \leqq t}\left|M_{\Delta}(s)\right|^{2}\right] \\
& \quad \leqq 4 \int_{S_{t}} E\left[\left|\bar{a}_{\Delta}(s, \theta, \phi, \alpha)-a(s, \theta, \phi, \alpha)\right|^{2}\right] \widehat{N}(d s d \theta d \phi d \alpha) \\
& \quad \leqq 16 \pi c \int_{0}^{t}\left\{E\left[\left|\bar{X}_{\Delta}(\Delta(s))-X(s)\right|^{2}\right]+I_{1, \Delta}(s)\right\} d s,
\end{aligned}
$$

and, by using Schwarz's inequality, we have

$$
\begin{aligned}
& E\left[\sup _{0 \leqq s \leqq t}\left|A_{\Delta}(s)\right|^{2}\right] \\
& =E\left[\sup _{0 \leqq s \leqq t} \mid \int_{s_{t}}\left\{\bar{X}_{\Delta}(\Delta(s))-X(s-)+Y(\Delta(s), \alpha)\right.\right. \\
& \left.\quad-Y(s-, \alpha)\}\left.\sin ^{2} \frac{\theta}{2} \hat{N}(d s d \theta d \phi d \alpha)\right|^{2}\right] \\
& \leqq c^{2} \pi^{2} t \int_{0}^{t}\left\{E\left[\left|\bar{X}_{\Delta}(\Delta(s))-X(s)\right|^{2}\right]+I_{1, \Delta}(s)\right\} d s .
\end{aligned}
$$

Since

$$
\bar{X}_{\Delta}(t)-X(t)=M_{\Delta}(t)+A_{\Delta}(t),
$$

setting

$$
I_{2, \Delta}(t)=E\left[\sup _{0 \leqq s \leqq t}\left|\bar{X}_{\Delta}(s)-X(s)\right|^{2}\right]
$$

we get

$$
I_{2, \Delta}(t) \leqq K \int_{0}^{t}\left\{I_{2, \Delta}(s)+E\left[|X(\Delta(s))-X(s)|^{2}\right]+I_{1, \Delta}(s)\right\} d s, t \leqq T,
$$

where $K \equiv K(T, c)=4\left(16 \pi c+c^{2} \pi^{2} T\right)$. Hence Gronwall's lemma shows that

$$
\begin{gathered}
I_{2, \Delta}(T) \leqq K e^{K T} \int_{0}^{T}\left\{E\left[|X(\Delta(s))-X(s)|^{2}\right]+I_{1, \Delta}(s)\right\} d s \\
\rightarrow 0 \quad \text { as }|\Delta| \rightarrow 0,
\end{gathered}
$$

which proves (3.19). Next, we show that, for every $T$ and $c_{0}>0$, there exists a positive constant $K_{3}$ such that

$$
\begin{aligned}
& \bar{\rho}_{t}^{2}\left(P(u(\cdot), \Delta), P\left(u^{\prime}(\cdot), \Delta\right)\right) \\
& \quad \leqq K_{3} \int_{0}^{t} \rho^{2}\left(u(\Delta(s)), u^{\prime}(\Delta(s))\right) d s, t \leqq T, c \in\left(0, c_{0}\right],
\end{aligned}
$$


for every partition $\Delta$ and $u(\cdot), u^{\prime}(\cdot) \in B\left([0, \infty), \mathscr{L}_{2}\right)$. In fact, we take two processes $Y(t, \alpha)$ and $Y^{\prime}(t, \alpha)$ on $\{(0,1), d \alpha\}$ in such a way that

(i) $Y(t, \cdot) \sim u(t), Y^{\prime}(t, \cdot) \sim u^{\prime}(t)$ for every $t \geqq 0$,

$\left\{\right.$ (ii) $\int_{0}^{1}\left|Y(t, \alpha)-Y^{\prime}(t, \alpha)\right|^{2} d \alpha=\rho^{2}\left(u(t), u^{\prime}(t)\right)$ for every $t \geqq 0$,

and define $X_{\Delta}(\cdot)$ by $(3.18)$ and $X_{\Delta}^{\prime}(\cdot)$ by

$$
\left\{\begin{array}{l}
X_{\Delta}^{\prime}(t)=X(0)+\int_{s_{t}} a_{\Delta}^{\prime}(s, \theta, \phi, \alpha) N(d s d \theta d \phi d \alpha) \\
a_{\Delta}^{\prime}(s, \theta, \phi, \alpha)=a\left(X_{\Delta}^{\prime}(\Delta(s)), Y^{\prime}(\Delta(s), \alpha), \theta, \phi\right. \\
\left.\quad+\phi_{0}\left(X_{\Delta}(\Delta(s)), Y(\Delta(s), \alpha), X_{\Delta}^{\prime}(\Delta(s)), Y^{\prime}(\Delta(s), \alpha)\right)\right) .
\end{array}\right.
$$

Then the probability distributions of $X_{\Delta}(\cdot)$ and $X_{\Delta}^{\prime}(\cdot)$ are $P(u(\cdot), \Delta)$ and $P\left(u^{\prime}(\cdot), \Delta\right)$, respectively. In a similar manner to bound $I_{2, \Delta}(t)$, we can show an estimate on $I_{3, \Delta}(t)=E\left[\sup _{0 \leqq s \leqq t}\left|X_{\Delta}(s)-X_{\Delta}^{\prime}(s)\right|^{2}\right]$ :

$$
I_{3, \Delta}(t) \leqq K(T, c) \int_{0}^{t}\left\{I_{3, \Delta}(s)+\rho^{2}\left(u(\Delta(s)), u^{\prime}(\Delta(s))\right)\right\} d s, t \leqq T,
$$

which implies (3.20) with the help of Gronwall's lemma. Tending $|\Delta|$ to 0 in both sides of (3.20), we get the desired estimate by noting (3.19).

Q.E.D.

Now, for the process $Y(t, \alpha)$ given by $(2.2)$, we set $Y_{T}(\alpha)=\sup _{0 \leqq t \leq T}$ $|Y(t, \alpha)|, T<\infty$, and consider a random variable $\bar{Y}_{T}(\alpha)$ on the probability space $\{(0,1), d \alpha\}$ satisfying the following two conditions.

$$
\bar{Y}_{T} \sim Y_{T} .
$$

$$
\bar{Y}_{T} \text { is non-decreasing in } \alpha \in(0,1) \text {. }
$$

Let $\Delta=\left\{\theta_{i}\right\}_{i=0}^{\infty}$ be a sequence such that $\pi \geqq \theta_{0}>\theta_{1}>\cdots>0$. For such $\Delta$, we set $B_{i}=\left(\theta_{i+1}, \theta_{i}\right], S^{i}=B_{i} \times(0,2 \pi) \times(0,1), i=0,1, \cdots$ and

$$
\begin{aligned}
& g(\alpha, \Delta) \equiv g(\alpha, \Delta ; Q, T, p) \\
& \quad=2 \pi \sum_{i=0}^{\infty} \theta_{i}^{p} Q\left(B_{i}\right) \exp \left\{-2 \pi T Q\left(B_{i}\right)(1-\alpha)\right\}, \alpha \in(0,1)
\end{aligned}
$$

Lemma 3.5. (i) Assume that there exists $\theta_{0} \in(0, \pi]$ such that $Q\left(\left[\theta_{0}, \pi\right]\right)=0$. Then we have 


$$
\begin{aligned}
E\left[\sup _{0 \leqq t \leqq T}|X(t)-X(t-)|^{p}\right] \leqq \theta_{0}^{p} E\left[\sup _{0 \leqq t \leqq T}|X(t)|^{p}\right] \\
\\
\quad+\inf _{\Delta} T \int_{0}^{1} \bar{Y}_{T}^{p}(\alpha) g(\alpha, \Delta) d \alpha,
\end{aligned}
$$

where the infimum is taken over all sequences $\Delta=\left\{\theta_{i}\right\}_{i=0}^{\infty}$.

(ii) The second term in the right hand side of the inequality in (i) is bounded by $2 \pi c T \theta_{0}^{p-2} \int_{0}^{1} \sup _{0 \leqq t \leqq T}|Y(t, \alpha)|^{p} d \alpha$.

Proof. Let $\{p(t, \omega), t \geqq 0\}=\{(\theta(t, \omega), \phi(t, \omega), \alpha(t, \omega)), t \geqq 0\}$ be a Poisson point process on $S$ corresponding to the Poisson random measure $N$, that is, $p(t)$ is an $S \cup\{\partial\}$-valued process such that $N(B)$ $=\sum_{t} 1_{B}(t, p(t))$ holds for every Borel subset $B$ of $(0, \infty) \times S$ satisfying $\widehat{N}(B)<\infty$, where $\partial$ is an extra point not belonging to $S$. Since the assumption on $Q$ implies that $P\left(\theta(t) \leqq \theta_{0}\right)=1$, we have

$$
\begin{aligned}
& \sup _{0 \leqq t \leqq T}|X(t)-X(t-)|^{p} \\
= & \sup _{0 \leqq t \leqq T, p(t) \in S}|a(X(t-), Y(t-, \alpha(t)), \theta(t), \phi(t))|^{p} \\
= & \sup _{0 \leqq t \leqq T, p(t) \in S}|X(t-)-Y(t-, \alpha(t))|^{p}\left\{\sin \frac{\theta(t)}{2}\right\}^{p} \\
\leqq & \theta_{0}^{p} \sup _{0 \leqq t \leqq T}|X(t)|^{p} \dashv \sup _{0 \leqq t \leqq T, p(t) \in S}\left(\theta_{0} \wedge \theta(t)\right){ }^{p} Y_{T}^{p}(\alpha(t)),(P \text {-a. s. }) .
\end{aligned}
$$

For a sequence $\Delta=\left\{\theta_{i}\right\}_{i=0}^{\infty}$, the following (3.25)-(3.27) are well-known (see, e.g., Ikeda and Watanabe [6]).

(3.25) The random variable $n^{i}(\omega) \equiv \sum_{0<t \leqq T} 1_{S^{i}}(p(t, \omega))$ is Poisson distributed with mean $2 \pi T Q\left(B_{i}\right)$ for each $i=0,1, \cdots$.

(3.26) Arranging the times $t$ which satisfy $p(t) \in S^{i}, 0<t<\infty$, in an increasing order; $0<t_{1}^{i}<t_{2}^{i}<\cdots$, random variables $\alpha_{k}^{i}(\omega) \equiv \alpha\left(t_{k}^{i}\right.$ $(\omega), \omega)$ are uniformly distributed on $(0,1)$.

(3.27) The random variables $n^{i}$ and $\alpha_{k}^{i}$, $i \geqq 0, k \geqq 1$, are mutually independent.

The final term in the right hand side of (3.24) is bounded as follows:

$$
I(t) \equiv \sup _{0 \leqq t \leqq T, p(t) \in S}\left(\theta_{0} \wedge \theta(t)\right)^{p} Y_{T}^{p}(\alpha(t))
$$




$$
\begin{aligned}
& \leqq \sum_{i=0}^{\infty} \theta_{i}^{p} \sup _{1 \leqq k \leqq n^{i}(\omega)} Y_{T}^{p}\left(\alpha_{k}^{i}(\omega)\right) \\
& \sim \sum_{i=0}^{\infty} \theta_{i}^{p} \sup _{1 \leqq k \leqq n^{i}(\omega)} \bar{Y}_{T}^{p}\left(\alpha_{k}^{i}(\omega)\right) \\
& =\sum_{i=0}^{\infty} \theta_{i}^{p} \bar{Y}_{T}^{p}\left(\sup _{1 \leqq k \leqq n^{i}(\omega)} \alpha_{k}^{i}(\omega)\right) .
\end{aligned}
$$

Noting that

$$
P\left(\sup _{1 \leqq k \leqq n} \alpha_{k}^{i}(\omega) \in d \alpha\right)=n \alpha^{n-1} d \alpha, 0 \leqq i, n<\infty
$$

we get

$$
\begin{aligned}
& E[I(T)] \\
& \quad \leqq \sum_{i=0}^{\infty} \theta_{i}^{p} \sum_{n=0}^{\infty} \frac{1}{n !}\left\{2 \pi T Q\left(B_{i}\right)\right\}^{n} \exp \left\{-2 \pi T Q\left(B_{i}\right)\right\} \int_{0}^{1} \bar{Y}_{T}^{p}(\alpha) n \alpha^{n-1} d \alpha \\
& \quad=T \int_{0}^{1} \bar{Y}_{T}^{p}(\alpha) g(\alpha, \Delta) d \alpha .
\end{aligned}
$$

Since this estimate holds for every $\Delta$, we obtain the assertion (i) by (3.24). The assertion (ii) follows immediately from $g(\alpha, \Delta)$ $\leqq 2 \pi \sum_{i=0}^{\infty} \theta_{i}^{p} Q\left(B_{i}\right)$ and $c=\inf _{\Delta} \sum_{i=0}^{\infty} \theta_{i}^{2} Q\left(B_{i}\right)$ Q.E.D.

\section{$\S 4$. Proof of the Main Theorem}

We now give the proof of Theorem 2.1. Throughout this section, the family $\left\{Q_{\varepsilon}\right\}$ is assumed to satisfy the assumption (I) if $f \in \mathscr{L}_{p}(p>2)$ or the assumption (II) if $f \in \mathscr{Q}_{2}$. We denote by $u^{\varepsilon}(t), 0<\varepsilon<1$, and $\bar{u}(t)$ the probability distributions of $X_{t}$ under $P_{f}^{\varepsilon}$ and $P_{f}$, respectively. For each $x \in R^{3}$, let $P_{f, x}^{\varepsilon}, 0<\varepsilon<1$, and $P_{f, x}$ be the probability distribution $P\left(\delta_{x}, u^{\varepsilon}(\cdot), \varepsilon^{-2} Q_{\varepsilon}\right)$ of the solution of (2.2) and that on the space $C$ of

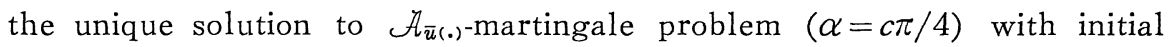
distribution $\delta_{x}$, respectively, where $\delta_{x}$ is the Dirac's $\delta$-measure with unit mass at $x$. We sometimes regard $P_{f, x}$ as a probability measure on ( $D$, $\mathscr{B}^{D}$ ). We show a strong version of Theorem 2.1.

Theorem 4. 1. The probability measures $P_{f}^{\varepsilon}$ and $P_{f, x}^{\varepsilon}$ converge to $P_{f}$ and $P_{f, x}$ as $\varepsilon \rightarrow 0$, respectively, in the following sense: 
$\lim _{\varepsilon \downarrow 0} \bar{\rho}_{T}\left(P_{f}^{\varepsilon}, P_{f}\right)=0$ and $\lim _{\varepsilon \downarrow 0} \bar{\rho}_{T}\left(P_{f, x}^{\varepsilon}, P_{f, x}\right)=0$ for every $T<\infty$.

Theorem 2.1 follows from Theorem 4.1 immediately since $\bar{\rho}$-convergence implies the weak convergence on the space $D_{S}$. The method to prove Theorem 4.1 is based on the martingale formulation which was used first by Stroock and Varadhan [18] and also by several authors (see, e.g., Bensoussan, Lions and Papanicolaou [2] and Papanicolaou, Stroock and Varadhan [15]) to show the weak convergence of Markov processes. In the following we set $c_{0}=\sup _{0<\varepsilon<1} c_{\varepsilon}<\infty$, where $c_{\varepsilon}$ is the number introduced in Section 2. We note that $c_{\varepsilon}=c$ for every $0<\varepsilon<1$ when the assumption (II) is fulfilled. We first note that the proof of Theorem 4.1 can be reduced to show the following lemma in which we set $\widetilde{P}_{f}^{\varepsilon}=P\left(f, \bar{u}(\cdot), \varepsilon^{-2} Q_{\varepsilon}\right) \quad$ and $\quad \widetilde{P}_{f, x}^{\varepsilon}=P\left(\delta_{x}, \bar{u}(\cdot), \varepsilon^{-2} Q_{\varepsilon}\right) \quad$ for $\quad 0<\varepsilon<1$ and $x \in \mathbb{R}^{3}$.

Lemma 4. 1. The probability measures $\widetilde{P}_{f}^{\varepsilon}$ and $\widetilde{P}_{f, x}^{\varepsilon}$ converge to $P_{f}$ and $P_{f, x}$ as $\varepsilon \rightarrow 0$, respectively, in the following sense:

$$
\lim _{\varepsilon \downarrow 0} \bar{\rho}_{T}\left(\widetilde{P}_{f}^{\varepsilon}, P_{f}\right)=0 \text { and } \lim _{\varepsilon \downarrow 0} \bar{\rho}_{T}\left(\widetilde{P}_{f, x}^{\varepsilon}, P_{f, x}\right)=0 \text { for every } T<\infty \text {. }
$$

Proof of Theorem 4.1. Using the triangle inequality for $\bar{\rho}_{t}$, since $u^{\varepsilon}(\cdot), \bar{u}(\cdot) \in \widetilde{C}\left([0, \infty), \mathscr{L}_{2}\right)$, Lemma 3.4 proves that

$$
\begin{aligned}
\bar{\rho}_{t}^{2}\left(P_{f}^{\varepsilon}, P_{f}\right) & \leqq 2 \bar{\rho}_{t}^{2}\left(\widetilde{P}_{f}^{\varepsilon}, P_{f}\right)+2 \bar{\rho}_{t}^{2}\left(P_{f}^{\varepsilon}, \widetilde{P}_{f}^{\varepsilon}\right) \\
& \leqq 2 \bar{\rho}_{T}^{2}\left(\widetilde{P}_{f}^{\varepsilon}, P_{f}\right)+2 K_{3}\left(T, c_{0}\right) \int_{0}^{t} \rho^{2}\left(u^{\varepsilon}(s), \bar{u}(s)\right) d s \\
& \leqq 2 \bar{\rho}_{T}^{2}\left(\widetilde{P}_{f}^{\varepsilon}, P_{f}\right)+2 K_{3}\left(T, c_{0}\right) \int_{0}^{t} \bar{\rho}_{s}^{2}\left(P_{f}^{\varepsilon}, P_{f}\right) d s, t \leqq T .
\end{aligned}
$$

Gronwall's lemma yields that

$$
\bar{\rho}_{T}^{2}\left(P_{f}^{\varepsilon}, P_{f}\right) \leqq 2 \bar{\rho}_{T}^{2}\left(\widetilde{P}_{f}^{\varepsilon}, P_{f}\right) \exp \left\{2 T K_{3}\left(T, c_{0}\right)\right\} .
$$

Since, by Lemma 4.1 , the right hand side tends to 0 as $\varepsilon \rightarrow 0$, we get $\lim _{\varepsilon \downarrow 0} \bar{\rho}_{T}^{2}\left(P_{f}^{\varepsilon}, P_{f}\right)=0$. The convergence of $P_{f, x}^{\varepsilon}$ can be shown as follows.

$$
\begin{aligned}
& \bar{\rho}_{T}^{2}\left(P_{f, x}^{\mathrm{\varepsilon}}, P_{f, x}\right) \leqq 2 \bar{\rho}_{T}^{2}\left(\widetilde{P}_{f, x}^{\varepsilon}, P_{f, x}\right)+2 \bar{\rho}_{T}^{2}\left(P_{f, x}^{\varepsilon}, \widetilde{P}_{f, x}^{\varepsilon}\right) \\
& \quad \leqq 2 \bar{\rho}_{T}^{2}\left(\widetilde{P}_{f, x}^{\varepsilon}, P_{f, x}\right)+2 K_{3}\left(T, c_{0}\right) \int_{0}^{T} \rho^{2}\left(u^{\varepsilon}(s), \bar{u}(s)\right) d s
\end{aligned}
$$


$\leqq 2 \bar{\rho}_{T}^{2}\left(\widetilde{P}_{f, x}^{\varepsilon}, P_{f, x}\right)+2 K_{3}\left(T, c_{0}\right) T \bar{\rho}_{T}^{2}\left(P_{f}^{\varepsilon}, P_{f}\right)$

$\rightarrow 0$ as $\varepsilon \rightarrow 0$.

Q.E.D.

As the first step to give a proof of Lemma 4.1, we show the tightness of the family $\left\{\widetilde{P}_{f, x}^{\varepsilon}\right\}$. To do this we note the following lemma whose proof is omitted since it is standard. For every $n=1,2, \cdots$, define a mapping $\Theta_{n}$ of $D$ by $\Theta_{n}(w)=w_{\tau_{n}}^{\cdot}$, weD, where $w_{\tau_{n}}^{\cdot}$ is a stopped path:

$$
w_{\tau_{n}}^{:}(t)=w\left(t \wedge \tau_{n}\right), \quad t \in[0, \infty),
$$

and

$$
\tau_{n}(w)=\inf \{t>0 ;|w(t)|>n\}
$$

Lemma 4. 2. Let $\left\{P^{\varepsilon} ; 0<\varepsilon<1\right\}$ be a family of probability measures on $\left(D, \mathcal{B}^{D}\right)$. If the family $\left\{P^{\varepsilon} \circ \Theta_{n}^{-1} ; 0<\varepsilon<1\right\}$ is relatively compact for every $n=1,2, \cdots$ and if

$$
\lim _{n \rightarrow \infty} \sup _{0<\varepsilon<1} P^{\varepsilon}\left(\tau_{n}<T\right)=0
$$

holds for every $T<\infty$, then the family $\left\{P^{\varepsilon} ; 0<\varepsilon<1\right\}$ is relatively compact.

Applying this lemma we get the following.

Lemma 4. 3. The family $\left\{\widetilde{P}_{f, x}^{\varepsilon} ; 0<\varepsilon<1\right\}$ is relatively compact.

Proof. We denote $\widetilde{P}_{f, x}^{\varepsilon}$ simply by $P^{\varepsilon}$. For every $n=1,2, \cdots$, Proposition 2.3-(iv) and Lemma 3.3-(iii) prove that

$$
\begin{aligned}
& E^{P^{\varepsilon_{0} \otimes_{n}-1}}\left[\left|X_{t_{3}}-X_{t_{2}}\right|^{2}\left|X_{t_{2}}-X_{t_{1}}\right|^{2}\right] \\
& \quad=E^{P^{\varepsilon}}\left[\left|X_{t_{3} \wedge \tau_{n}}-X_{t_{2} \wedge \tau_{n}}\right|^{2}\left|X_{t_{2} \wedge \tau_{n}}-X_{t_{1} \wedge \tau_{n}}\right|^{2}\right] \\
& \quad \leqq K_{2}\left(T, c_{0}\right)^{2}\left\{n^{2}+\|f\|^{2}\right\}^{2}\left(t_{3}-t_{1}\right)^{2}, 0 \leqq t_{1} \leqq t_{2} \leqq t_{3} \leqq T .
\end{aligned}
$$

This estimate implies the relative compactness of the family $\left\{P^{\varepsilon} \circ \Theta_{n}^{-1}\right.$; $0<\varepsilon<1\}$ for every $n=1,2, \cdots$ (Billingsley [3]). While, by Lemma 3.3(i) with $p=2$, we have

$$
P^{\varepsilon}\left(\tau_{n} \leqq T\right)=P^{\varepsilon}\left(\sup _{0 \leqq t \leqq T}\left|X_{t}\right|>n\right)
$$




$$
\begin{aligned}
& \leqq n^{-2} E^{P^{\varepsilon}}\left[\sup _{0 \leqq t \leqq T}\left|X_{t}\right|^{2}\right] \\
& \leqq n^{-2} K_{1}\left(T,\|f\|, c_{0}, 2\right)\left\{1+|x|^{2}\right\},
\end{aligned}
$$

which proves that $\lim _{n \rightarrow \infty} \sup _{0<\varepsilon<1} P^{\varepsilon}\left(\tau_{n}<T\right)=0$ for each $T<\infty$. Hence Lemma 4.2 shows the conclusion.

Q.E.D.

We give a criterion to prove the $\bar{\rho}$-convergence.

Lemma 4. 4. Let $\left\{P^{\varepsilon} ; 0 \leqq \varepsilon<1\right\}$ be a family of probability measures on $\left(D, \mathscr{B}^{D}\right)$ satisfying the following three conditions:

(4.1) $P^{\varepsilon}$ tends weakly to $P^{0}$ on the space $D_{S}$ as $\varepsilon \rightarrow 0$.

$$
\begin{gathered}
P^{0}(C)=1 . \\
\lim _{n \rightarrow \infty} \sup _{0<\varepsilon<1} E^{P^{\varepsilon}}\left[\sup _{0 \leqq t \leqq T}\left|X_{t}\right|^{q} ; \sup _{0 \leqq t \leqq T}\left|X_{t}\right|>n\right]=0, \quad q \geqq 2 .
\end{gathered}
$$

Then we have $\lim _{\varepsilon \downarrow 0} \bar{\rho}_{q, T}\left(P^{\varepsilon}, P^{0}\right)=0$.

Proof. By the theorem of Skorohod (see, e.g., Ikeda and Watanabe [6]), the condition (4.1) shows that there exists a probability space $(\Omega, \mathscr{I}, P)$ and $D$-valued random variables $\left\{X^{\varepsilon}(\cdot), 0 \leqq \varepsilon<1\right\}$ defined on $\Omega$ which satisfy the following two conditions.

(4.4) For each $\varepsilon \in[0,1)$, the probability distribution of $X^{\varepsilon}$ is $P^{\varepsilon}$.

(4.5) $X^{\varepsilon}$ converges to $X^{0}$ in the space $D_{S}$ as $\varepsilon \rightarrow 0$ (P-a.s.).

Since the condition (4.2) implies that $P\left(X^{0} \in C\right)=1$, the condition (4.5) shows the following (see Billingsley [3]).

(4.6) $X^{\varepsilon}$ converges to $X^{0}$ in the space $D_{U}$ as $\varepsilon \rightarrow 0$ (P-a.s.).

Now we see that

$$
\begin{aligned}
& \bar{\rho}_{q, T}^{q}\left(P^{\varepsilon}, P^{0}\right) \leqq E^{P}\left[\sup _{0 \leqq t \leqq T}\left|X^{\varepsilon}(t)-X^{0}(t)\right|^{q}\right] \\
& \leqq E^{P}\left[\sup _{0 \leqq t \leqq T}\left|X^{\varepsilon}(t)-X^{0}(t)\right|^{q} \wedge n\right] \\
& \quad+E^{P}\left[\sup _{0 \leqq t \leqq T}\left|X^{\varepsilon}(t)-X^{0}(t)\right|^{q} ; \sup _{0 \leqq t \leqq T}\left|X^{\varepsilon}(t)-X^{0}(t)\right|^{q} \geqq n\right] \\
& \equiv I_{1, n}^{\varepsilon}+I_{2, n}^{\varepsilon} \text { for every } n<\infty .
\end{aligned}
$$

The condition (4.6) proves that $\lim _{\varepsilon \rightarrow 0} I_{1, n}^{\varepsilon}=0$. We can also show that $\lim _{n \rightarrow \infty} \sup _{0<\varepsilon<1} I_{2, n}^{\varepsilon}=0$ by noting the condition (4.3). Hence we obtain the conclusion.

Q.E.D. 
Lemma 4.5. (i) The families $\left\{P_{f, x}^{\varepsilon} ; 0<\varepsilon<1\right\}$ and $\left\{\widetilde{P}_{f, x}^{\varepsilon} ; 0<\varepsilon\right.$ $<1\}$ satisfy the condition (4.3) for every $q \geqq 2$.

(ii) The families $\left\{P_{f}^{\varepsilon} ; 0<\varepsilon<1\right\}$ and $\left\{\widetilde{P}_{f}^{\varepsilon} ; 0<\varepsilon<1\right\}$ satisfy the condition (4.3) with $q=p$.

Proof. By Proposition 2.2-(iii) and Lemma 3.3-(i), we have

$$
\begin{aligned}
& E^{P_{f, x}^{\varepsilon}}\left[\sup _{0 \leqq t \leqq T}\left|X_{t}\right|^{q} ; \sup _{0 \leqq t \leqq T}\left|X_{t}\right|>n\right] \\
& \leqq\left\{E^{P_{f, x}^{\varepsilon}}\left[\sup _{0 \leqq t \leqq T}\left|X_{t}\right|^{2 q}\right]\right\}^{1 / 2}\left\{P_{f, x}^{\varepsilon}\left[\sup _{0 \leqq t \leqq T}\left|X_{t}\right|>n\right]\right\}^{1 / 2} \\
& \leqq\left\{E^{P_{f, x}^{\varepsilon}}\left[\sup _{0 \leqq t \leqq T}\left|X_{t}\right|^{2 q}\right]\right\}^{1 / 2}\left\{n^{-4 q} E^{P_{f, x}^{\varepsilon}}\left[\sup _{0 \leqq t \leqq T}\left|X_{t}\right|^{4 q}\right]\right\}^{1 / 2} \\
& \leqq n^{-2 q}\left[K_{1}\left(T,\|f\|, c_{0}, 2 q\right)\left\{|x|^{2 q}+1\right\} K_{1}\left(T,\|f\|, c_{0}, 4 q\right)\left\{|x|^{4 q}+1\right\}\right]^{1 / 2} \\
& \leqq C n^{-2 q}\left(|x|^{3 q}+1\right) \rightarrow 0 \text { as } n \rightarrow \infty \text { (uniformly in } \varepsilon \text { ), }
\end{aligned}
$$

where $C \equiv C\left(T,\|f\|, c_{0}, q\right)$ is a positive constant. Hence we get the assertion (i) for the family $\left\{P_{f, x}^{\varepsilon}\right\}$. Noting the relation $P_{f}^{\varepsilon}(\cdot)=\int_{\boldsymbol{R}^{3}} P_{f, x}^{\varepsilon}(\cdot) f(d x)$, we have, by (4.7) with $q=p$,

$$
\begin{aligned}
& E^{P_{f}^{\varepsilon}}\left[\sup _{0 \leqq t \leqq T}\left|X_{t}\right|^{p} ; \sup _{0 \leqq t \leqq T}\left|X_{t}\right|>n\right] \\
& \leqq E^{P_{f}^{\varepsilon}}\left[\sup _{0 \leqq t \leqq T}\left|X_{t}\right|^{p} ;\left|X_{0}\right| \geqq \sqrt{n}\right] \\
&+E^{P_{f}^{\varepsilon}}\left[\sup _{0 \leqq t \leqq T}\left|X_{t}\right|^{p} ; \sup _{0 \leqq t \leqq T}\left|X_{t}\right|>n,\left|X_{0}\right|<\sqrt{n}\right] \\
&= \int_{|x| \geqq \sqrt{n}} E^{P_{f, x}^{\varepsilon}}\left[\sup _{0 \leq t \leq T}\left|X_{t}\right|^{p}\right] f(d x) \\
&+\int_{|x|<\sqrt{n}} E^{P_{f, x}^{\varepsilon}}\left[\sup _{0 \leqq t \leqq T}\left|X_{t}\right|^{p} ; \sup _{0 \leqq t \leqq T}\left|X_{t}\right|>n\right] f(d x) \\
& \leqq K_{1}\left(T,\|f\|, c_{0}, p\right) \int_{|x| \geqq \sqrt{n}}\left\{|x|^{p}+1\right\} f(d x)+C n^{-2 p}\left(n^{3 p / 2}+1\right) \\
& \rightarrow 0 \text { as } n \rightarrow \infty \text { (uniformly in } \varepsilon \text { ), }
\end{aligned}
$$

which proves the assertion (ii) for the family $\left\{P_{f}^{\varepsilon}\right\}$. In a similar manner, we can also show the assertions (i) and (ii) for the families $\left\{\widetilde{P}_{f, x}^{\varepsilon}\right\}$ and $\left\{\widetilde{P}_{f}^{\varepsilon}\right\}$, respectively.

Q.E.D. 
For $u \in \mathscr{L}_{2}$, we put

$\mathcal{A}_{u}^{\varepsilon} \psi(x)=\int_{(0, \pi) \times(0,2 \pi) \times \mathbb{R}^{3}}\left\{\psi\left(x^{*}\right)-\psi(x)\right\} \varepsilon^{-2} Q_{\varepsilon}(d \theta) d \phi u(d y), \phi \in C_{0}^{\infty}\left(\mathbb{R}^{3}\right)$.

The following lemma can be shown by using Itô's formula relative to the Poisson stochastic integrals.

Lemma 4. 6. For each $\psi \in C_{0}^{\infty}\left(\mathbb{R}^{3}\right), \psi\left(X_{t}\right)-\int_{0}^{t} \mathfrak{A}_{s}^{\varepsilon} \psi\left(X_{s}\right) d s, t \geqq 0$, is a martingale relative to $\left(\widetilde{P}_{f, x}^{\varepsilon},\left\{\mathscr{B}_{t}^{D} ; t \geqq 0\right\}\right)$, where $\mathcal{A}_{s}^{\varepsilon}=\mathcal{A}_{\bar{u}(s)}^{\varepsilon}$.

We show the uniform boundedness and the equicontinuity of the functions $\left\{\mathcal{A}_{t}^{\varepsilon} \psi(x) ; 0<\varepsilon<1 / 2, t \in[0, \infty)\right\}$.

Lemma 4. 7. (i) For each $\psi \in C_{0}^{\infty}\left(\mathbb{R}^{3}\right)$, we have

$$
\sup _{(\varepsilon, t, x) \in(0,1 / 2) \times[0, \infty) \times \boldsymbol{R}^{3}}\left|\mathcal{A}_{t}^{\varepsilon} \psi(x)\right|<\infty .
$$

(ii) For each $\psi \in C_{0}^{\infty}\left(\mathbb{R}^{3}\right)$, we have

$$
\begin{aligned}
& \sup _{(\varepsilon, t) \in(0,1) \times[0, \infty)}\left|\mathcal{A}_{t}^{\varepsilon} \psi(x)-\mathcal{A}_{t}^{\varepsilon} \psi\left(x^{\prime}\right)\right| \\
& \leqq K(\psi) c_{0}\left\{1+|x|^{2}+\left|x^{\prime}\right|^{2}+\|f\|^{2}\right\}\left|x-x^{\prime}\right|,
\end{aligned}
$$

where $K(\psi)$ is the constant appeared in Lemma 3.2.

Proof. Since

$$
\mathcal{A}_{t}^{\varepsilon} \psi(x)=\int_{(0, \pi) \times \boldsymbol{R}^{3}} G(x, y, \theta ; \psi) \varepsilon^{-2} Q_{\varepsilon}(d \theta) \bar{u}(t, d y),
$$

Proposition 2.3-(iv) and Lemma 3.2-(i), (ii) show that

$$
\left|\mathcal{A}_{t}^{\varepsilon} \psi(x)\right| \leqq K(\psi) c_{0}\left\{1+|x|^{2}+\|f\|^{2}\right\}
$$

and

$$
\left|\mathscr{A}_{t}^{\varepsilon} \psi(x)-\mathscr{A}_{t}^{\varepsilon} \psi\left(x^{\prime}\right)\right| \leqq K(\psi) c_{0}\left\{1+|x|^{2}+\left|x^{\prime}\right|^{2}+\|f\|^{2}\right\}\left|x-x^{\prime}\right| \text {. }
$$

The inequality (4.9) implies the assertion (ii). Lemma 3.2-(iii) proves, for $\varepsilon \in(0,1 / 2)$ and $x:|x| \geqq 3 M \equiv 3 M(\psi) \quad(>0)$, that

$$
\left|\mathscr{A}_{t}^{\varepsilon} \psi(x)\right|=\left|\int_{0}^{\pi} \varepsilon^{-2} Q_{\varepsilon}(d \theta) \int_{|y|>M / 3 \theta} \bar{u}(t, d y) \int_{0}^{2 \pi} \psi\left(x^{*}\right) d \phi\right|
$$




$$
\begin{aligned}
& \leqq 2 \pi \sup _{x^{\prime}}\left|\psi\left(x^{\prime}\right)\right| \int_{0}^{\pi} \bar{u}(t ;\{|y|>M / 3 \theta\}) \varepsilon^{-2} Q_{\varepsilon}(d \theta) \\
& \leqq 18 \pi c_{0}\|f\|^{2} M^{-2} \sup _{x^{\prime}}\left|\psi\left(x^{\prime}\right)\right| .
\end{aligned}
$$

Combining this with (4.8), we get the assertion (i). Q.E.D.

We prove the convergence of the function $\mathscr{A}_{u}^{\varepsilon} \psi(x)$ as $\varepsilon \rightarrow 0$.

Lemma 4. 8. For each $u \in \mathscr{L}_{2}, x \in \boldsymbol{R}^{3}$ and $\psi \in C_{0}^{\infty}\left(\boldsymbol{R}^{3}\right)$, the function $\mathcal{A}_{u}^{\varepsilon} \psi(x)$ converges to $\mathcal{A}_{u} \psi(x)$ as $\varepsilon \rightarrow 0$, where $\mathcal{A}_{u}$ is the operator defined in Section 1 with $\alpha=c \pi / 4$.

Proof. We divide the proof into three steps.

Step 1. For each $x \in \boldsymbol{R}^{3}$, we consider a region

$$
\boldsymbol{K}_{x}=\left\{(\theta, y) \in(0, \pi) \times \boldsymbol{R}^{3} ;|x-y| \sin \frac{\theta}{2} \geqq|x|+M\right\},
$$

where $M \equiv M(\psi)$ is the constant defined in Lemma 3.2. The relation $\left|x-x^{*}\right|=|x-y| \sin \frac{\theta}{2}$ shows that $\psi\left(x^{*}\right)=0$ if $(\theta, y) \in \boldsymbol{K}_{x}$. Therefore we have

$$
\begin{gathered}
G(x, y, \theta ; \psi) \\
=\left\{\begin{array}{cc}
\langle\nabla \psi(x), \boldsymbol{b}(x, y, \theta)\rangle+\frac{1}{2} \sum_{i, j=1}^{3} \boldsymbol{a}_{i j}(x, y, \theta) \frac{\partial^{2} \psi}{\partial x_{i} \partial x_{j}}(x)+R, \\
-2 \pi \psi(x), & (\theta, y) \notin \boldsymbol{K}_{x}, \\
& (\theta, y) \in \boldsymbol{K}_{x},
\end{array}\right.
\end{gathered}
$$

where

$$
\begin{aligned}
& \boldsymbol{a}_{i j}(x, y, \theta)=\int_{0}^{2 \pi}\left(x_{i}^{*}-x_{i}\right)\left(x_{j}^{*}-x_{j}\right) d \phi, 1 \leqq i, j \leqq 3, \\
& \boldsymbol{b}(x, y, \theta)=2 \pi(y-x) \sin ^{2} \frac{\theta}{2}
\end{aligned}
$$

and

$$
\begin{aligned}
R & \equiv R(x, y, \theta) \\
& =\frac{1}{6} \sum_{i, j, k=1}^{3} \int_{0}^{2 \pi} \frac{\partial^{3} \psi}{\partial x_{i} \partial x_{j} \partial x_{k}}\left(x^{0}\right)\left(x_{i}^{*}-x_{i}\right)\left(x_{j}^{*}-x_{j}\right)\left(x_{k}^{*}-x_{k}\right) d \phi,
\end{aligned}
$$


with some $x^{0} \in \boldsymbol{R}^{3}$ which depends on $x, y, \theta, \phi$ and $\psi$.

Step 2. We show that

(4.10) $\lim _{\varepsilon \downarrow 0} \int_{\boldsymbol{K}_{x}}\left|\boldsymbol{a}_{i j}(x, y, \theta)\right| \varepsilon^{-2} Q_{\varepsilon}(d \theta) u(d y)=0,1 \leqq i, j \leqq 3$,

and

(4. 11) $\lim _{\varepsilon \downarrow 0} \int_{\boldsymbol{K}_{x}}|\boldsymbol{b}(x, y, \theta)| \varepsilon^{-2} Q_{\varepsilon}(d \theta) u(d y)=0$.

By noting (3.3), the equality (4.10) is proved in the following manner:

$$
\begin{aligned}
& \int_{\boldsymbol{K}_{x}}\left|\boldsymbol{a}_{i j}(x, y, \theta)\right| \varepsilon^{-2} Q_{\varepsilon}(d \theta) u(d y) \\
& \quad \leqq \frac{\pi}{2} \int_{\boldsymbol{K}_{x}} \theta^{2}|x-y|^{2} \varepsilon^{-2} Q_{\varepsilon}(d \theta) u(d y) \\
& \leqq \int_{0}^{\pi} \theta^{2} g_{1}(x, \theta) \varepsilon^{-2} Q_{\varepsilon}(d \theta) \\
& \leqq c_{0} \sup _{0<\theta \leq \varepsilon \pi} g_{1}(x, \theta)=c_{0} g_{1}(x, \varepsilon \pi) \rightarrow 0 \quad(\varepsilon \rightarrow 0),
\end{aligned}
$$

where

$$
g_{1}(x, \theta)=\frac{\pi}{2} \int_{|y|>(|x|+M)\{\sin (\theta / 2)\}-1}|y|^{2} u_{x}(d y)
$$

and the probability measure $u_{x}$ is defined by $u_{x}(B)=u(B+x)$ for every Borel subset $B$ of $\boldsymbol{R}^{3}$. The equality (4.11) is shown by

$$
\begin{aligned}
\int_{\boldsymbol{K}_{x}}|\boldsymbol{b}(x, y, \theta)| \varepsilon^{-2} Q_{\varepsilon}(d \theta) u(d y) \\
\leqq \\
\leqq \frac{\pi}{2} \int_{\boldsymbol{K}_{x}} \theta^{2}|y-x| \varepsilon^{-2} Q_{\varepsilon}(d \theta) u(d y) \\
\leqq c_{0}\left\{\pi g_{1}(x, \varepsilon \pi) / 2\right\}^{1 / 2} \rightarrow 0 \quad(\varepsilon \rightarrow 0)
\end{aligned}
$$

The next task is to show that

$$
\text { (4.12) } \lim _{\varepsilon \downarrow 0} \int_{\boldsymbol{K}_{x}} \varepsilon^{-2} Q_{\varepsilon}(d \theta) u(d y)=0
$$

and

$$
\text { (4. 13) } \lim _{\varepsilon \downarrow 0} \int_{\boldsymbol{K}_{x^{c}}}|R(x, y, \theta)| \varepsilon^{-2} Q_{\varepsilon}(d \theta) u(d y)=0 \quad\left(\mathbb{K}_{x}^{c}=(0, \pi) \times \mathbb{R}^{3}-\mathbb{K}_{x}\right) \text {. }
$$


To prove these equalities, we note that the following two equalities hold for every $v \in \mathscr{L}_{2}$.

$$
\begin{aligned}
& \lim _{n \rightarrow \infty} n^{2} v(|x|>n)=0, \\
& \lim _{n \rightarrow \infty} \frac{1}{n} \int_{|x|<n}|x|^{3} v(d x)=0 .
\end{aligned}
$$

To show (4.12), we see that

$$
\begin{aligned}
\int_{K_{x}} \varepsilon^{-2} Q_{\varepsilon}(d \theta) u(d y) \\
\quad=\int_{0}^{\pi} u_{x}\left(\left\{y ;|y| \sin \frac{\theta}{2} \geqq|x|+M\right\}\right) \varepsilon^{-2} Q_{\varepsilon}(d \theta) \\
\quad=\int_{0}^{\pi} g_{2}(x, \theta) \theta^{2} \varepsilon^{-2} Q_{\varepsilon}(d \theta) \\
\quad \leqq c_{0} \sup _{0<\theta \leqq \varepsilon \pi} g_{2}(x, \theta)
\end{aligned}
$$

where

$$
g_{2}(x, \theta)=\theta^{-2} u_{x}\left(\left\{y ;|y| \sin \frac{\theta}{2} \geqq|x|+M\right\}\right) .
$$

Since (4.14) yields that $\lim _{\varepsilon \downarrow 0} \sup _{0<\theta \leqq \varepsilon \pi} g_{2}(x, \theta)=0$, we obtain (4.12). To prove (4.13), noting that the relation $\left|x^{*}-x\right|=|x-y| \sin \frac{\theta}{2}$ shows

$$
|R| \leqq 4 K \theta^{3}|x-y|^{3}, K=\sup _{i, j, k, x}\left|\frac{\partial^{3} \psi}{\partial x_{i} \partial x_{j} \partial x_{k}}(x)\right|,
$$

we have

$$
\begin{aligned}
& \int_{\boldsymbol{K}_{x^{c}}}|R(x, y, \theta)| \varepsilon^{-2} Q_{\varepsilon}(d \theta) u(d y) \\
& \leqq 4 K \int_{K_{x^{c}}} \theta^{3}|x-y|^{3} \varepsilon^{-2} Q_{\varepsilon}(d \theta) u(d y) \\
& \leqq 4 K \int_{0}^{\pi} \theta^{2} g_{3}(x, \theta) \varepsilon^{-2} Q_{\varepsilon}(d \theta) \leqq 4 K c_{0} \sup _{0<\theta \leqq \varepsilon \pi} g_{3}(x, \theta),
\end{aligned}
$$

where

$$
g_{3}(x, \theta)=\theta \int_{|y|<(|x|+M)\{\sin (\theta / 2)\}-1}|y|^{3} u_{x}(d y) .
$$

Since (4.15) implies that $\lim _{\varepsilon \downarrow 0} \sup _{0<\theta \leq \varepsilon \pi} g_{3}(x, \theta)=0$, we obtain (4.13). 
Step 3. The four equalities (4.10)-(4.13) show that the proof is completed if the following two equalities are shown.

(4. 16) $\lim _{\varepsilon \downarrow 0} \int_{(0, \pi) \times \boldsymbol{R}^{3}} \boldsymbol{a}_{i j}(x, y, \theta) \varepsilon^{-2} Q_{\varepsilon}(d \theta) u(d y)=\boldsymbol{a}_{i j}(x, u), 1 \leqq i, j \leqq 3$, and

(4. 17) $\lim _{\varepsilon \downarrow 0} \int_{(0, \pi) \times \boldsymbol{R}^{3}} \boldsymbol{b}(x, y, \theta) \varepsilon^{-2} Q_{\varepsilon}(d \theta) u(d y)=b(x, u)$,

where $\boldsymbol{a}_{i j}(x, u)$ and $\boldsymbol{b}(x, u)$ are the functions defined in Section 1 (we take $\alpha=c \pi / 4)$. Since (4.17) is shown easily, we give the proof of (4.16). First we note that

$$
\begin{aligned}
\boldsymbol{a}_{i j}(x, y, \theta)= & 2 \pi\left(y_{i}-x_{i}\right)\left(y_{j}-x_{j}\right) \sin ^{4} \frac{\theta}{2} \\
& +\frac{|x-y|^{2}}{4} \sin ^{2} \theta \int_{0}^{2 \pi} e_{i}(\phi) e_{j}(\phi) d \phi, \quad 1 \leqq i, j \leqq 3,
\end{aligned}
$$

where $e_{i}(\phi)$ is an $i$-th component of the vector $e(\phi)$. Therefore we get

$$
\text { (4.18) } \begin{aligned}
& \lim _{\varepsilon \downarrow 0} \int_{(0, \pi) \times \boldsymbol{R}^{3}} \boldsymbol{a}_{i j}(x, y, \theta) \varepsilon^{-2} Q_{\varepsilon}(d \theta) u(d y) \\
& \quad=\frac{c}{4} \int_{\boldsymbol{R}^{3}}|x-y|^{2} u(d y) \int_{0}^{2 \pi} e_{i}(\phi) e_{j}(\phi) d \phi, \quad 1 \leqq i, j \leqq 3 .
\end{aligned}
$$

The final task is to compute the integral in the right hand side of (4.18). We have to remind that the function $e(\phi)$ depends also on $x$ and $y$. We put $z=\left(z_{i}\right)_{i=1}^{3}=(x-y) /|x-y|$ (assuming $\left.x \neq y\right)$ and $f(\phi)=\left(f_{1}(\phi)\right.$, $\left.f_{2}(\phi), f_{3}(\phi)\right)=(\cos \phi, \sin \phi, 0) \in \mathbb{R}^{3}$. By introducing a matrix $T=$ $\left(T_{i j}\right)_{1 \leqq i, j \leqq 3}$ defined by

$$
\text { (i) } T=\bar{z}^{-1}\left(\begin{array}{ccc}
z_{1} z_{3}, & -z_{2}, & \bar{z} z_{1} \\
z_{2} z_{3}, & z_{1}, & \bar{z} z_{2} \\
-\bar{z}^{2}, & 0, & \bar{z} z_{3}
\end{array}\right) \quad \text { if } \quad \bar{z}=\left\{z_{1}^{2}+z_{2}^{2}\right\}^{1 / 2}>0,
$$

and

$$
\text { (ii) } T=\left(\begin{array}{lll}
z_{3}, & 0, & 0 \\
0, & 1, & 0 \\
0, & 0, & z_{3}
\end{array}\right) \text { if } \quad \bar{z}=0
$$

we see that 


$$
e_{i}(\phi)=\sum_{j=1}^{3} T_{i j} f_{j}\left(\phi+\phi_{0}\right), \quad 1 \leqq i \leqq 3,
$$

with some $\phi_{0} \in[0,2 \pi)$. Therefore the equality

$$
\int_{0}^{2 \pi} f_{i}(\phi) f_{j}(\phi) d \phi=\delta_{i j}\left(1-\delta_{i 3}\right) \pi, \quad 1 \leqq i, j \leqq 3,
$$

yields that

$$
\begin{aligned}
\int_{0}^{2 \pi} e_{i}(\phi) e_{j}(\phi) d \phi & =\sum_{k_{1}, k_{2}} T_{i k_{1}} T_{j k_{2}} \int_{0}^{2 \pi} f_{k_{1}}(\phi) f_{k_{2}}(\phi) d \phi \\
& =\pi \sum_{k=1}^{2} T_{i k} T_{j k}, \quad 1 \leqq i, j \leqq 3
\end{aligned}
$$

Since the right hand side of this equality is given by

$$
\left(\sum_{k=1}^{2} T_{i k} T_{j k}\right)_{1 \leqq i, j \leqq 3}=\left(\begin{array}{ccc}
1-z_{1}^{2}, & -z_{1} z_{2}, & -z_{1} z_{3} \\
-z_{1} z_{2}, & 1-z_{2}^{2}, & -z_{2} z_{3} \\
-z_{1} z_{3}, & -z_{2} z_{3}, & 1-z_{3}^{2}
\end{array}\right),
$$

we have that

$$
\begin{gathered}
|x-y|^{2} \int_{0}^{2 \pi} e_{i}(\phi) e_{j}(\phi) d \phi=\pi\left\{\delta_{i j}|x-y|^{2}-\left(x_{i}-y_{i}\right)\left(x_{j}-y_{j}\right)\right\} \\
1 \leqq i, j \leqq 3
\end{gathered}
$$

Substituting this equality into the right hand side of (4.18), we obtain (4.16) and therefore we get the conclusion.

Q.E.D.

We are now ready to prove Lemma 4.1 .

Proof of Lemma 4.1. The proof will be given in four steps.

Step 1. To complete the proof, by Lemma 4.4 and Lemma 4.5, it is sufficient only to show that $\widetilde{P}_{f}^{\varepsilon}$ and $\widetilde{P}_{f, x}^{\varepsilon}$ converge weakly to $P_{f}$ and $P_{f, x}$, respectively. But the weak convergence of $\left\{\widetilde{P}_{f}^{\varepsilon}\right\}$ follows from that of $\left\{\widetilde{P}_{f, x}^{\varepsilon}\right\}$ for every $x \in \boldsymbol{R}^{3}$. Since Lemma 4.3 implies that each subsequence $\left\{\widetilde{P}_{f, x}^{\varepsilon^{\prime}}\right\}$ of $\left\{\widetilde{P}_{f, x}^{\varepsilon}\right\}$ contains a further subsequence $\left\{\widetilde{P}_{f, x}^{\varepsilon^{\prime \prime}}\right\}$ which converges weakly to some probability distribution $P$, it proves the conclusion that $P=P_{f, x}$. For simplicity, we denote the subsequence $\left\{\widetilde{P}_{f, x}^{\varepsilon^{\prime \prime}}\right\}$ by $\left\{P^{\varepsilon}\right\}$.

Step 2. Here we show that $P(C)=1$. To this end, we first prove 
that

(4. 19) $\quad \lim _{\varepsilon \downarrow 0} I_{\varepsilon} \equiv \lim _{\varepsilon \downarrow 0} E^{P^{\varepsilon}}\left[\sup _{0 \leqq t \leqq T}\left|X_{t}-X_{t-}\right|^{p}\right]=0$ for every $T<\infty$.

When $p>2$ (i.e., $f \in \mathscr{Q}_{p}, p>2$ ), taking $\{Y(t, \alpha) ; t \geqq 0\}$ to be a $P_{f^{-}}$ distributed stochastic process on $\{(0,1), d \alpha\}$, Lemma 3.5 gives an estimate:

$$
I_{\varepsilon} \leqq(\varepsilon \pi)^{p} E^{P^{\varepsilon}}\left[\sup _{0 \leqq t \leqq T}\left|X_{t}\right|^{p}\right]+2 \pi c_{0} T(\varepsilon \pi)^{p-2} E^{P_{f}}\left[\sup _{0 \leqq t \leqq T}\left|X_{t}\right|^{p}\right] .
$$

Since Lemma 3.3-(i) shows that $\sup _{0<\varepsilon<1} E^{P^{\varepsilon}}\left[\sup _{0 \leqq t \leqq T}\left|X_{t}\right|^{p}\right]<\infty$ and Proposition 2.3-(iii) shows that $E^{P_{f}}\left[\sup _{0 \leqq t \leqq T}\left|X_{t}\right|^{p}\right]<\infty$, we get (4.19). Now we prove (4.19) when $p=2$ (i.e., $f \in \mathscr{L}_{2}$ ). For a given sequence $\Delta=\left\{\theta_{i}\right\}_{i=0}^{\infty}$ with $\left\{B_{i}\right\}_{i=0}^{\infty}$ such that $\theta_{0}=\varepsilon \pi$, we introduce a new sequence $\Delta^{\prime}=\left\{\theta_{i}^{\prime}\right\}_{i=0}^{\infty}$ with $\theta_{i}^{\prime}=\varepsilon^{-1} \theta_{i}$ and $B_{i}^{\prime}=\varepsilon^{-1} B_{i}$. Then, by the assumption (II), we have

$$
\begin{aligned}
g\left(\alpha, \Delta ; \varepsilon^{-2} Q_{\varepsilon}, T, 2\right) \\
\quad=\sum_{i=0}^{\infty} 2 \pi \theta_{i}^{2} \varepsilon^{-2} Q_{\varepsilon}\left(B_{i}\right) \exp \left\{-2 \pi T \varepsilon^{-2} Q_{\varepsilon}\left(B_{i}\right)(1-\alpha)\right\} \\
\quad=\sum_{i=0}^{\infty} 2 \pi\left(\theta_{i}^{\prime}\right)^{2} Q\left(B_{i}^{\prime}\right) \exp \left\{-2 \pi T \varepsilon^{-2} Q\left(B_{i}^{\prime}\right)(1-\alpha)\right\} \\
\quad=g\left(\alpha, \Delta^{\prime} ; Q, \varepsilon^{-2} T, 2\right),
\end{aligned}
$$

where $g$ is the function introduced by (3.23). Hence Lemma 3.5 shows that

$$
I_{\varepsilon} \leqq(\varepsilon \pi)^{2} E^{P^{\varepsilon}}\left[\sup _{0 \leqq t \leqq T}\left|X_{t}\right|^{2}\right]+\inf _{\Delta ; \theta_{0}=\pi} K_{\varepsilon}(\Delta)
$$

where

$$
K_{\varepsilon}(\Delta)=\sum_{i=0 ; Q\left(B_{i}\right)>0}^{\infty} 2 \pi T \theta_{i}^{2} Q\left(B_{i}\right) \int_{0}^{1} \bar{Y}_{T}^{2}(\alpha) \exp \left\{-2 \pi T \varepsilon^{-2} Q\left(B_{i}\right)(1-\alpha)\right\} d \alpha .
$$

We note that $\bar{Y}_{T}(\cdot)$ is a random variable defined on the probability space $\{(0,1), d \alpha\}$ with the same distribution as $\sup _{0 \leqq t \leqq T}\left|X_{t}\right|$ under $P_{f}$. Since the measure $Q$ satisfies that $\int_{0}^{\pi} \theta^{2} Q(d \theta)<\infty$, we can take a sequence $\bar{\Delta}=\left\{\bar{\theta}_{i}\right\}_{i=0}^{\infty}$ with $\left\{\bar{B}_{i}\right\}_{i=0}^{\infty}$ which satisfies $\bar{\theta}_{0}=\pi$ and $\sum_{i=0}^{\infty} \bar{\theta}_{i}^{2} Q\left(\bar{B}_{i}\right)<\infty$. Noting that

$$
\sum_{i=0}^{\infty} \bar{\theta}_{i}^{2} Q\left(\bar{B}_{i}\right) \int_{0}^{1} \bar{Y}_{T}^{2}(\alpha) d \alpha<\infty
$$


Lebesgue's dominated convergence theorem proves that $\lim _{\varepsilon \downarrow 0} K_{\varepsilon}(\bar{J})=0$ and accordingly that $\lim _{\varepsilon \downarrow 0} I_{\varepsilon}=0$. Therefore the equality (4.19) has been proved for every $p \geqq 2$.

Since the function $\sup _{0 \leq t<T}\left|X_{t}(w)-X_{t-}(w)\right|$, weD, is lower semi-continuous on the space $D_{S}$, the set $\left\{w \in D ; \sup _{0 \leq t<T}\left|X_{t}(w)-X_{t-}(w)\right|>\lambda\right\}$ is open in $D_{S}$ for every $\lambda>0$. Hence, noting that $P^{\varepsilon}$ tends weakly to $P$ as $\varepsilon \rightarrow 0$ on $D_{S}$, we obtain that

$$
\begin{aligned}
& P\left(\sup _{0 \leqq t<T}\left|X_{t}-X_{t-}\right|>\lambda\right) \leqq \frac{\lim _{\varepsilon \downarrow 0}}{P^{\varepsilon}}\left(\sup _{0 \leqq t<T}\left|X_{t}-X_{t-}\right|>\lambda\right) \\
& \leqq \lim _{\varepsilon \downarrow 0} \lambda^{-p} I_{\varepsilon}=0 \text { for every } T<\infty \text { and } \lambda>0 \text {, }
\end{aligned}
$$

which proves that $P(C)=1$.

Step 3. We show the following.

$$
\left\{\begin{array}{l}
\text { For each } \phi \in C_{0}^{\infty}\left(\boldsymbol{R}^{3}\right), \psi\left(X_{t}\right)-\int_{0}^{t} \mathscr{A}_{\bar{u}(\boldsymbol{s})} \psi\left(X_{s}\right) d s, t \geqq 0, \text { is a } \\
\text { martingale relative to }\left(P,\left\{\mathscr{B}_{t}^{D} ; t \geqq 0\right\}\right) .
\end{array}\right.
$$

We set

$$
\eta(t)=\psi\left(X_{t}\right)-\int_{0}^{t} \mathcal{A}_{\bar{u}(s)} \psi\left(X_{s}\right) d s
$$

and

$$
\eta^{\varepsilon}(t)=\psi\left(X_{t}\right)-\int_{0}^{t} \mathcal{A}_{s}^{\varepsilon} \psi\left(X_{s}\right) d s, \quad t \geqq 0 .
$$

To prove (4.20), it is enough to show that

$$
E^{P}\left[\eta\left(t^{\prime}\right) \Phi\right]=E^{P}[\eta(t) \Phi], t^{\prime} \geqq t \geqq 0,
$$

for every $\mathscr{B}_{t}^{D}$-measurable $\Phi \in C_{b}\left(D_{S}\right)$ where $C_{b}\left(D_{S}\right)$ is the space of real valued bounded continuous functions on $D_{S}$. Since Lemma 4.6 shows that $E^{P^{\varepsilon}}\left[\eta^{\varepsilon}\left(t^{\prime}\right) \Phi\right]=E^{P^{\varepsilon}}\left[\eta^{\varepsilon}(t) \Phi\right]$, (4.21) follows from

(4.22) $\lim _{\varepsilon \downarrow 0} E^{P^{\varepsilon}}\left[\eta^{\varepsilon}(t) \Phi\right]=E^{P}[\eta(t) \Phi]$ for every $t \geqq 0$ and $\Phi \in C_{b}\left(D_{S}\right)$.

To prove $(4.22)$, it is sufficient to show that

$$
\lim _{\varepsilon \downarrow 0} E^{P^{\varepsilon}}\left[\psi\left(X_{t}\right) \Phi\right]=E^{P}\left[\psi\left(X_{t}\right) \Phi\right]
$$

and 


$$
\lim _{\varepsilon \downarrow 0} E^{P^{\varepsilon}}\left[\Psi^{\varepsilon} \cdot \Phi\right]=E^{P}[\Psi \cdot \Phi],
$$

where

$$
\Psi^{\varepsilon}(w)=\int_{0}^{t} \mathcal{A}_{s}^{\varepsilon} \psi\left(X_{s}(w)\right) d s \text { and } \Psi(w)=\int_{0}^{t} \mathcal{A}_{\bar{u}(s)} \psi\left(X_{s}(w)\right) d s, w \in D .
$$

Since $P(C)=1$ and $P^{\varepsilon}$ tends weakly to $P$ on $D_{S}$, we see that

$$
\lim _{\varepsilon \downarrow 0} E^{P^{\varepsilon}}[\widetilde{\Phi}]=E^{P}[\widetilde{\Phi}]
$$

for every $\mathscr{B}^{D}$-measurable $\widetilde{\Phi} \in C_{b}\left(D_{U}\right)$, where $C_{b}\left(D_{U}\right)$ is the space of real valued bounded continuous functions on $D_{U}$. Therefore we get (4.23) by noting that $\psi\left(X_{t}\right) \in C_{b}\left(D_{U}\right)$. While Lemma 4.7 shows that $\left\{\Psi^{\varepsilon}(w)\right.$; $0<\varepsilon<1 / 2\}$ is a family of uniformly bounded functions which are equicontinuous relative to the $S$-topology at every $w \in D$. Lemma 4.8 shows that $\Psi^{\varepsilon}(w)$ tends to $\Psi(w)$ as $\varepsilon \rightarrow 0$ for each $w \in D$. Hence AscoliArzela's theorem proves that $\Psi^{\varepsilon}$ tends to $\Psi$ uniformly on each compact subset of $D_{S}$ so that we can show (4.24) in a usual manner and this concludes the proof of (4.20).

Step 4. We have shown that the probability distribution $P$ is a

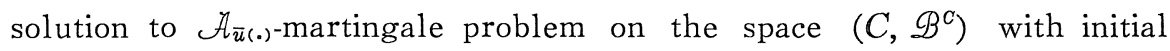
distribution $\delta_{x}$. The uniqueness of the solution to the martingale problem implies that $P=P_{f, x}$ which completes the proof. Q.E.D.

As an immediate consequence of Theorem 4.1 and Lemma 4.5, we obtain the following.

Corollary 4. 1. For every $T<\infty$ and $q \geqq 2$, we have

$$
\lim _{\varepsilon \downarrow 0} \bar{\rho}_{p, T}\left(P_{f}^{\varepsilon}, P_{f}\right)=0 \quad \text { and } \quad \lim _{\varepsilon \downarrow 0} \bar{\rho}_{q, T}\left(P_{f, x}^{\varepsilon}, P_{f, x}\right)=0
$$

Let $e_{f}^{\varepsilon}(t, x) \equiv e_{f}^{\varepsilon}(t, x, \cdot)$ and $e_{f}(t, x) \equiv e_{f}(t, x, \cdot), 0<\varepsilon<1, \quad t \geqq 0, x$ $\in \mathbb{R}^{3}$, be probability distributions of $X_{t}$ under $P_{f, x}^{\varepsilon}$ and $P_{f, x}$, respectively. These $e_{f}^{\varepsilon}$ and $e_{f}$ are called transition functions associated with (1.4) and (1.6), respectively (see Tanaka [20]). Another consequence of Theorem 4.1 is the convergences of the nonlinear semigroup and the transition function. 
Corollary 4.2. (i) $A s \varepsilon \rightarrow 0, u^{\varepsilon}(\cdot)$ tends to $\bar{u}(\cdot)$ in the space $C\left([0, \infty), \mathscr{L}_{p}\right)$, that is, we have that

$$
\lim _{\varepsilon \downarrow 0} \sup _{0 \leqq t \leqq T} \rho_{p}\left(u^{\varepsilon}(t), \bar{u}(t)\right)=0 \text { for every } T<\infty .
$$

(ii) For every $q \geqq 2$, as $\varepsilon \rightarrow 0, e_{f}^{\varepsilon}(\cdot, x)$ tends to $e_{f}(\cdot, x)$ in the space $C\left([0, \infty), \mathscr{L}_{q}\right)$, that is, we have that

$$
\lim _{\varepsilon \downarrow 0} \sup _{0 \leqq t \leqq T} \rho_{q}\left(e_{f}^{\varepsilon}(t, x), e_{f}(t, x)\right)=0 \text { for every } T<\infty .
$$

Proof. Noting that

$$
\sup _{0 \leq t \leq T} \rho_{p}\left(u^{\varepsilon}(t), \bar{u}(t)\right) \leqq \bar{\rho}_{p, T}\left(P_{f}^{\varepsilon}, P_{f}\right)
$$

and

$$
\sup _{0 \leqq t \leqq T} \rho_{q}\left(e_{f}^{\varepsilon}(t, x), e_{f}(t, x)\right) \leqq \bar{\rho}_{q, T}\left(P_{f, x}^{\varepsilon}, P_{f, x}\right),
$$

we get the conclusion by Corollary 4.1.

Q.E.D.

\section{$\S$ 5. Properties of Associated Diffusion Process with Landau Equation}

We study (1) the time evolution of moments and (2) the trend to the equilibrium for the associated diffusion process with Landau equation (1.6). Let $\mathcal{A}_{u}, u \in \mathscr{Q}_{2}$, be the differential operator introduced in Section 1 and let $P \equiv P_{f}$ be the unique solution to the martingale problem (2.3) with initial distribution $f \in \mathscr{Q}_{2}$. We denote by $\bar{u}(t) \equiv U_{t} f$ the distribution of $X_{t}$ under $P$.

(1) Time evolution of moments. Ikenberry and Truesdell [7] studied the time evolution of moments for solutions to Boltzmann equation of Maxwellian molecules. Their method is available in our case so that, for each integer $k \geqq 0$, we choose $2 k+1$ linearly independent homogeneous harmonic polynomials $\left\{\xi_{k}^{l}(x) ;|l| \leqq k\right\}$ of degree $k$ in $\boldsymbol{R}^{3}$ (see Tanaka [20]). We put

$$
\begin{aligned}
& \xi_{n}(x)=|x|^{2 r} \xi_{k}^{l}(x) \text { for } \boldsymbol{n}=(r, k, l) \\
& \xi_{(0,0,0)}(x)=1
\end{aligned}
$$


where $r=0,1, \cdots, k=0,1, \cdots$, and $|l| \leqq k$. The degree of $\xi_{n}$ is $|\boldsymbol{n}|=2 r$ $+k$. Then every homogeneous polynomial of $x$ with degree $n$ can be expressed by a linear combination of $\xi_{n}(x)$ with $|\boldsymbol{n}|=n$. We discuss the time evolution of harmonic moments:

$$
\mu_{n}(t)=E^{P}\left[\xi_{n}\left(X_{t}\right)\right] .
$$

Note that (2.4) determines $\mu_{\boldsymbol{n}}(t)$ for $|\boldsymbol{n}|=1,2$ completely.

Theorem 5.1. If the initial distribution $f$ belongs to the space $\mathscr{L}_{p}$ with $p \geqq 3$, then, for every $n ; 3 \leqq|n| \leqq p$, we have

$$
\frac{d}{d t} \mu_{\boldsymbol{n}}(t)=\sum^{\prime} \alpha \cdot \beta_{\boldsymbol{n}_{1}, \boldsymbol{n}_{2}}^{\boldsymbol{n}} \mu_{\boldsymbol{n}_{1}}(t) \mu_{\boldsymbol{n}_{2}}(t)-\frac{\alpha}{2}\{2|\boldsymbol{n}|+k(k+1)\} \mu_{\boldsymbol{n}}(t),
$$

with some constants $\beta_{\boldsymbol{n}_{1}, n_{2}}^{\boldsymbol{n}}$ independent of $\alpha$, where $\Sigma^{\prime}$ means the summation taken over all pairs $\left(\boldsymbol{n}_{1}, \boldsymbol{n}_{2}\right)$ satisfying $\left|\boldsymbol{n}_{1}\right|+\left|\boldsymbol{n}_{2}\right|=|\boldsymbol{n}|$ and $\left|\boldsymbol{n}_{1}\right|=1,2$.

Proof. First we note that (2.4) and (2.5) show that

$$
\begin{aligned}
& \boldsymbol{a}_{i j}(x, \bar{u}(t))-\boldsymbol{a}_{i j}(x)= \alpha\left\{\delta_{i j}\left(\|f\|^{2}-2\langle x, m(f)\rangle\right)+x_{i} m_{j}(f)\right. \\
&\left.+x_{j} m_{i}(f)-E^{P}\left[X_{i}(t) X_{j}(t)\right]\right\}, 1 \leqq i, j \leqq 3, \\
& \boldsymbol{b}(x, \bar{u}(t))-\boldsymbol{b}(x)=2 \alpha m(f) .
\end{aligned}
$$

Hence $\mathcal{A}_{\bar{u}(t)} \xi_{n}(x)-\mathcal{A}_{\boldsymbol{n}}(x)$ can be expressed as a summation $\Sigma^{\prime} \alpha$. $\beta_{\boldsymbol{n}_{1}, \boldsymbol{n}_{2}}^{\boldsymbol{n}} \mu_{\boldsymbol{n}_{1}}(t) \xi_{\boldsymbol{n}_{2}}(x)$ with some constants $\beta_{\boldsymbol{n}_{1}, \boldsymbol{n}_{2}}^{\boldsymbol{n}}$, where $\mathcal{A}$ is an operator defined by

$$
\mathcal{A}=\frac{1}{2} \sum_{i, j=1}^{3} \boldsymbol{a}_{i j}(x) \frac{\partial^{2}}{\partial x_{i} \partial x_{j}}+\sum_{i=1}^{3} \boldsymbol{b}_{i}(x) \frac{\partial}{\partial x_{i}} .
$$

Therefore two equalities

$$
\mathcal{A} \xi_{n}(x)=-\frac{\alpha}{2}\{2|\boldsymbol{n}|+k(k+1)\} \xi_{n}(x)
$$

and

$$
\frac{d}{d t} \mu_{n}(t)=E^{P}\left[\mathcal{A}_{\bar{u}(t)} \xi_{n}\left(X_{t}\right)\right]
$$

prove the conclusion.

Q.E.D. 
Remark. In the quantum mechanics (see, e.g., Landau and Lifshitz [11]), the operator of the angular momentum vector is given by

$$
\boldsymbol{L}=-i\left(x_{2} \frac{\partial}{\partial x_{3}}-x_{3} \frac{\partial}{\partial x_{2}}, x_{3} \frac{\partial}{\partial x_{1}}-x_{1} \frac{\partial}{\partial x_{3}}, x_{1} \frac{\partial}{\partial x_{2}}-x_{2} \frac{\partial}{\partial x_{1}}\right) .
$$

The operator $\mathcal{A}$ appeared in the proof of Theorem 5.1 can be represented in terms of $\boldsymbol{L}$ :

$$
\mathcal{A}=-\frac{\alpha}{2} \sum_{i=1}^{3} L_{i}^{2}-\alpha \sum_{i=1}^{3} x_{i} \frac{\partial}{\partial x_{i}}
$$

where $L_{i}$ is the $i$-th component of $\mathbb{L}$.

(2) Trend to the equilibrium. Let $g \equiv g_{f}$ be the Gaussian distribution on $\boldsymbol{R}^{3}$ defined as in Section 2-(1).

Lemma 5. 1. The distribution $g$ is invariant under $U_{t}$.

Proof. By (2.4) and (2.5), we see that $\mathcal{A}_{U^{g}}=\mathcal{A}_{g}$ for every $t \geqq 0$. But an easy calculation shows that $\int \mathcal{A}_{g} \psi(x) g(d x)=0$ for every $\psi \in$ $C_{0}^{\infty}\left(\boldsymbol{R}^{3}\right)$ and this proves the conclusion.

Q.E.D.

Lemma 5.2. Assume that the distribution $f$ belongs to the space $\mathscr{L}_{\infty} \equiv \underset{p \geqq 2}{\cap} \mathscr{Q}_{p}$. Then $U_{t} f$ converges weakly to $g$ as $t \rightarrow \infty$.

Proof. By Theorem 5.1, Lemma 5.1 and (2.4), we can prove that $\mu_{n}(t)$ converges to $\int \xi_{n}(x) g(d x)$ exponentially fast as $t \rightarrow \infty$ for every $\boldsymbol{n}$ (see the corollary to Theorem 8.1 of Tanaka [20]). Therefore we have the conclusion.

Q.E.D.

The following theorem is shown by using results for the Boltzmann equation of Maxwellian molecules.

Theorem 5.2. (i) The nonlinear semigroup $\left\{U_{t}\right\}$ on $\mathscr{Q}_{2}$ is nonexpansive with respect to the metric $\rho$ :

$$
\rho\left(U_{t} f_{1}, U_{t} f_{2}\right) \leqq \rho\left(f_{1}, f_{2}\right), \quad t \geqq 0, \quad f_{1}, f_{2} \in \mathscr{Q}_{2} .
$$


(ii) As $t \rightarrow \infty, \boldsymbol{e}\left(U_{t} f\right)$ decreases to 0 , where $\boldsymbol{e}$ is the functional introduced in Section 2-(1).

Proof. (i) Take a family $\left\{Q_{\varepsilon} ; 0<\varepsilon<1\right\}$ of Borel measures on $(0, \pi)$ satisfying the assumption (II) of Section 2-(4) with $Q ; \int_{0}^{\pi} \theta^{2} Q(d \theta)$ $=4 \alpha / \pi$. Then, by solving the stochastic differential equation (2.8), we get a family $\left\{\left\{T_{t}^{\varepsilon} ; t \geqq 0\right\} ; 0<\varepsilon<1\right\}$ of nonlinear semigroups. Corollary 4.2 shows that $\lim _{\varepsilon \downarrow 0} \rho\left(T_{t}^{\varepsilon} f, U_{t} f\right)=0$ for each $t \geqq 0$ and $f \in \mathscr{Q}_{2}$. While Proposition 2.2-(iv) shows that $\rho\left(T_{t}^{\varepsilon} f_{1}, T_{t}^{\varepsilon} f_{2}\right) \leqq \rho\left(f_{1}, f_{2}\right)$ for every $\varepsilon ; 0$ $<\varepsilon<1$. Hence we obtain the assertion (i) by using the triangle inequality for $\rho$ :

$$
\begin{aligned}
& \rho\left(U_{t} f_{1}, U_{t} f_{2}\right) \\
& \quad \leqq \lim _{\varepsilon \downarrow 0}\left\{\rho\left(T_{t}^{\varepsilon} f_{1}, U_{t} f_{1}\right)+\rho\left(T_{t}^{\varepsilon} f_{1}, T_{t}^{\varepsilon} f_{2}\right)+\rho\left(T_{t}^{\varepsilon} f_{2}, U_{t} f_{2}\right)\right\} \leqq \rho\left(f_{1}, f_{2}\right) .
\end{aligned}
$$

(ii) The decreasing property of $\boldsymbol{e}\left(U_{t} f\right)$ in $t$ follows from (i). For $f \in \mathscr{L}_{\infty}$, we get the conclusion by Lemma 5.2 noting that the family $\left\{U_{t} f ; t \geqq 0\right\}$ is $L^{2}$-uniformly integrable on $\mathbb{R}^{3}$. For general $f \in \mathscr{L}_{2}(v(f)$ $>0)$, we can choose $f_{\varepsilon} \in \mathscr{L}_{\infty}$ satisfying that $m\left(f_{\varepsilon}\right)=m(f), v\left(f_{\varepsilon}\right)=v(f)$ and $\rho\left(f, f_{\varepsilon}\right)<\varepsilon$. By using (i), we can complete the proof. Q.E.D.

\section{$\S$ 6. One-Dimensional Model}

We consider the following one-dimensional analogue of the Boltzmann equation of Maxwellian molecules:

$$
\begin{gathered}
\frac{\partial u}{\partial t}(t, x)=\int_{(-\pi, \pi) \times \boldsymbol{R}^{1}}\left\{u\left(t, x^{*}\right) u\left(t, y^{*}\right)-u(t, x) u(t, y)\right\} Q(d \theta) d y, \\
(t, x) \in(0, \infty) \times \mathbb{R}^{1}, \\
x^{*}=x \cos \theta-y \sin \theta, \quad y^{*}=x \sin \theta+y \cos \theta,
\end{gathered}
$$

where $Q$ is a Borel measure on $(-\pi, \pi)$ satisfying $\int_{-\pi}^{\pi}|\theta| Q(d \theta)<\infty$. Kac [8] discussed the equation (6.1) when $Q(d \theta)=d \theta / 2 \pi$. We set $S$ $=(-\pi, \pi) \times(0,1), S_{t}=(0, t] \times S$ and $a(x, y, \theta)=x^{*}-x$. Let $N \equiv N(d s$ $d \theta d \alpha)$ be a Poisson random measure on $(0, \infty) \times S$ with intensity measure $d s Q(d \theta) d \alpha$ defined on a probability space $(\Omega, \mathscr{I}, P)$ and let $f$ be a 
probability distribution on $\boldsymbol{R}^{1}$ satisfying $\|f\|^{2} \equiv \int_{\boldsymbol{R}^{1}} x^{2} f(d x)<\infty,\|f\|>0$. Similarly to the three-dimensional case, we can associate a one-dimensional Markov process $X(t)$ with the weak version of (6.1) by solving the following stochastic differential equation (see Murata [14]):

$$
\left\{\begin{array}{l}
X(t)=X(0)+\int_{s_{t}} a\left(X\left(s^{-}\right), Y\left(s^{-}, \alpha\right), \theta\right) N(d s d \theta d \alpha) \\
X(t) \sim Y(t, \cdot) \quad \text { for every } t \geqq 0 \\
X(0) \sim f,
\end{array}\right.
$$

where $X(t) \sim Y(t, \cdot)$ and $X(0) \sim f$ mean the equivalence in law.

Our objective here is to discuss the scaling limit of the equation (6.1). We define a family $\left\{Q_{\varepsilon} ; 0<\varepsilon<1\right\}$ of Borel measures on ( $-\pi$, $\pi)$ by $Q_{\varepsilon}(B)=Q\left(\varepsilon^{-1} B \cap(-\pi, \pi)\right)$ for every Borel subset $B$ of $(-\pi$, $\pi)$ and consider the following two types of scalings:

(I) $t \rightarrow \varepsilon^{-1} t, Q \rightarrow Q_{\varepsilon}$.

(II) $t \rightarrow \varepsilon^{-2} t, \quad Q \rightarrow Q_{\varepsilon}$.

Scaling (I). We set $b=\int_{-\pi}^{\pi} \theta Q(d \theta)$. After the transformation (I), we have a Markov process $X^{\varepsilon}(t)$ which is given by the following stochastic differential equation:

$$
\left\{\begin{array}{l}
X^{\varepsilon}(t)=X(0)+\int_{S_{t}} a\left(X^{\varepsilon}(s-), Y^{\varepsilon}(s-, \alpha), \theta\right) N_{\varepsilon}(d s d \theta d \alpha) \\
X^{\varepsilon}(t) \sim Y^{\varepsilon}(t, \cdot) \quad \text { for each } t \geqq 0 \\
X(0) \sim f,
\end{array}\right.
$$

where $N_{\varepsilon}$ is a Poisson random measure on $(0, \infty) \times S$ with intensity measure $\widehat{N}_{\varepsilon}=\varepsilon^{-1} d s Q_{\varepsilon}(d \theta) d \alpha$. We show that, as $\varepsilon \rightarrow 0, X^{\varepsilon}(t)$ tends to a stochastic process $X(t)$ defined by

$$
X(t)=X(0)+\left(e^{-b t}-1\right) \int_{R^{1}} x f(d x)
$$

Theorem 6.1. For every $T<\infty$, we have

$$
\lim _{\varepsilon \downarrow 0} E\left[\sup _{0 \leqq t \leqq T}\left\{X^{\varepsilon}(t)-X(t)\right\}^{2}\right]=0 .
$$

Proof. We set

$$
M^{\varepsilon}(t)=\int_{s_{t}} a\left(X^{\varepsilon}(s-), Y^{\varepsilon}(s-, \alpha), \theta\right) \tilde{N}_{\varepsilon}(d s d \theta d \alpha)
$$


and

$$
A^{\varepsilon}(t)=\int_{s_{t}} a\left(X^{\varepsilon}\left(s^{-}\right), Y^{\varepsilon}(s-, \alpha), \theta\right) \widehat{N}_{\varepsilon}(d s d \theta d \alpha),
$$

where $\widetilde{N}_{\varepsilon}=N_{\varepsilon}-\widehat{N}_{\varepsilon}$. First we show that

(6.4) $\sup _{0<\varepsilon<1} E\left[\sup _{0 \leqq t \leqq T}\left\{X^{\varepsilon}(t)\right\}^{2}\right]<\infty$ for every $T<\infty$.

In fact, Doob's inequality shows that

$$
\begin{gathered}
E\left[\sup _{0 \leqq s \leqq t}\left\{M^{\varepsilon}(s)\right\}^{2}\right] \leqq 4 E\left[\left\{M^{\varepsilon}(t)\right\}^{2}\right] \\
\quad=4 \int_{s_{t}} E\left[a^{2}\left(X^{\varepsilon}(s-), Y^{\varepsilon}(s-, \alpha), \theta\right)\right] \widehat{N}_{\varepsilon}(d s d \theta d \alpha) \\
\quad \leqq 32 \varepsilon \int_{-\pi}^{\pi} \theta^{2} Q(d \theta) \int_{0}^{t} E\left[\left\{X^{\varepsilon}(s)\right\}^{2}\right] d s
\end{gathered}
$$

While we have

$$
\begin{aligned}
& \leqq E\left[\left\{\int_{s_{t}}\left|a\left(X^{\varepsilon}(s-), Y^{\varepsilon}(s-, \alpha), \theta\right)\right| \widehat{N}_{\varepsilon}(d s d \theta d \alpha)\right\}^{2}\right] \\
& \leqq E\left[\int_{S_{t}}|\theta| \widehat{N}_{\varepsilon}(d s d \theta d \alpha) \int_{S_{t}}|\theta|\left\{\pi\left|X^{\varepsilon}\left(s^{-}\right)\right| / 2+\left|Y^{\varepsilon}\left(s^{-}, \alpha\right)\right|\right\}^{2} \widehat{N}_{\varepsilon}(d s d \theta d \alpha)\right] \\
& \leqq 8 t\left\{\int_{-\pi}^{\pi}|\theta| Q(d \theta)\right\}^{2} \int_{0}^{t} E\left[\left\{X^{\varepsilon}(s)\right\}^{2}\right] d s .
\end{aligned}
$$

Combining two estimates (6.5) and (6.6) and applying Gronwall's lemma, we get (6.4). In this procedure, although we do not know in advance that $E\left[\sup _{0 \leqq s \leqq t}\left\{X^{\varepsilon}(s)\right\}^{2}\right]<\infty$ for each $\varepsilon$, we can apply Gronwall's lemma properly by using cut-off technique which was employed in the proof of Lemma 3.3-(i). Noting that $X(t)$ satisfies an equation:

$$
X(t)=X(0)-b \int_{0}^{t} E[X(s)] d s,
$$

we get

$$
\begin{aligned}
& E\left[\sup _{0 \leqq s \leqq t}\left\{X(0)+A^{\varepsilon}(s)-X(s)\right\}^{2}\right] \\
= & E\left[\left\{\int_{0}^{t}\left|\int_{S} a\left(X^{\varepsilon}(s-), Y^{\varepsilon}(s-, \alpha), \theta\right) \varepsilon^{-1} Q_{\varepsilon}(d \theta) d \alpha-b E[X(s)]\right| d s\right\}^{2}\right]
\end{aligned}
$$




$$
\begin{aligned}
& \leqq t E\left[\int_{0}^{t}\left\{\left|X^{\varepsilon}(s)\right| \frac{\varepsilon}{2} \int_{-\pi}^{\pi} \theta^{2} Q(d \theta)+\left|b_{\varepsilon} E\left[X^{\varepsilon}(s)\right]-b E[X(s)]\right|\right\}^{2} d s\right] \\
& \leqq C_{1} t\left\{\left(\varepsilon^{2}+\left|b_{\varepsilon}-b\right|^{2}\right) \int_{0}^{t} E\left[\left\{X^{\varepsilon}(s)\right\}^{2}\right] d s+b^{2} \int_{0}^{t} E\left[\left\{X^{\varepsilon}(s)-X(s)\right\}^{2}\right] d s\right\},
\end{aligned}
$$

where

$$
b_{\varepsilon}=\int_{-\pi}^{\pi} \sin \theta \varepsilon^{-1} Q_{\varepsilon}(d \theta)
$$

and $C_{1}$ is a positive constant independent of $\varepsilon$. This estimate combined with (6.5) shows that

$$
\begin{aligned}
& E\left[\sup _{0 \leqq s \leqq t}\left\{X^{\varepsilon}(s)-X(s)\right\}^{2}\right] \\
& \leqq C_{2}\left(\varepsilon+\left|b_{\varepsilon}-b\right|^{2}\right) \int_{0}^{T} E\left[\left\{X^{\varepsilon}(s)\right\}^{2}\right] d s+C_{2} \int_{0}^{t} E\left[\sup _{0 \leqq r \leqq s}\left\{X^{\varepsilon}(r)-X(r)\right\}^{2}\right] d s, \\
& t \leqq T, 0<\varepsilon<1,
\end{aligned}
$$

with some positive constant $C_{2} \equiv C_{2}(T)$. Hence Gronwall's lemma yields that

$$
\begin{gathered}
E\left[\sup _{0 \leqq t \leqq T}\left\{X^{\varepsilon}(t)-X(t)\right\}^{2}\right] \leqq C_{2} e^{C_{2} T}\left(\varepsilon+\left|b_{\varepsilon}-b\right|^{2}\right) \int_{0}^{T} E\left[\left\{X^{\varepsilon}(s)\right\}^{2}\right] d s, \\
0<\varepsilon<1,
\end{gathered}
$$

which implies the conclusion noting (6.4) and that $\lim _{\varepsilon \downarrow 0}\left|b_{\varepsilon}-b\right|=0$.

Q.E.D.

Scaling (II). We assume the centering condition: $\int_{-\pi}^{\pi} \theta Q(d \theta)=0$ and set $c=\int_{-\pi}^{\pi} \theta^{2} Q(d \theta)$. After the transformation (II), we have a Markov process $X^{\varepsilon}(t)$ which is an integrable solution of the stochastic differential equation (6.3) with a Poisson random measure $N_{\varepsilon}$ on $(0, \infty) \times S$ whose intensity measure is now given by $\varepsilon^{-2} d s Q_{\varepsilon}(d \theta) d \alpha$. We denote by $P_{f}^{\varepsilon}$ the probability distribution on the space $D\left([0, \infty), \boldsymbol{R}^{1}\right)$ of $X^{\varepsilon}(\cdot)$. Let $P_{f}$ be the probability distribution on the space $C\left([0, \infty), \mathbb{R}^{1}\right)$ of $X(\cdot)$ which is a solution of the following stochastic differential equation:

$$
d X(t)=\|f\| \sqrt{c} d w(t)-c X(t) / 2 d t, \quad t \geqq 0,
$$

with initial distribution $f$ where $w(t)$ is a one-dimensional Brownian mo- 
tion. We can show the following theorem by applying a similar method used for the three-dimensional problem with much simplification so that we omit the proof.

Theorem 6.2. The probability measure $P_{f}^{\varepsilon}$ converges weakly to $P_{f}$ on the space $D\left([0, \infty), \mathbb{R}^{1}\right)$ as $\varepsilon \rightarrow 0$.

The following one-dimensional analogues of the results in Section 5 can be shown easily.

(i) Conservation of energy holds, that is, we have $E^{P_{f}}\left[\{X(t)\}^{2}\right]$ $=\|f\|^{2}$ for every $t \geqq 0$.

(ii) The distribution of $X(t)$ tends to the Gaussian distribution with mean 0 and variance $\|f\|^{2}$ as $t \rightarrow \infty$.

\section{References}

[1] Balescu, R., Equilibrium and nonequilibrium statistical mechanics, John Wiley and Sons, New York 1975.

[2] Bensoussan, A., Lions, J. L. and Papanicolaou, G. C., Boundary layers and homogenization of transport processes, Publ. RIMS, Kyoto Univ., 15 (1979), 53-157.

[3] Billingsley, P., Convergence of probability measures, John Wiley and Sons, New York 1968.

[4] Dellacherie, C. et Meyer, P. A., Probabilités et Potentiel (édition entièrement refondue), Herman, Paris 1980.

[5] Funaki, T., A certain class of diffusion processes associated with non-linear parabolic equations, preprint.

[6] Ikeda, N. and Watanabe, S., Stochastic differential equations and diffusion processes, North-Holland/Kodansha, Amsterdam/Tokyo 1981.

[7] Ikenberry, E. and Truesdell, C., On the pressure and the flux of energy in a gas according to Maxwell's kinetic theory I, J. Rational Mech. Anal., 5 (1956), 1-54.

[8] Kac, M., Foundations of kinetic theory, Proc. Third Berkeley Symp. on Math. Stat. and Prob., III, 171-197, Univ. of Calif. Press, Berkeley and Los Angeles, 1956.

[9] Kazamaki, N., Theory of martingale, Seminar on Probability 51, 1981 (in Japanese).

[10] Kurtz, T. G., Semigroups of conditioned shifts and approximation of Markov processes, Ann. Probability, 3 (1975), 618-642.

[11] Landau, L. D. and Lifshitz, E. M., Quantum mechanics (second edition, English translation), Pergamon Press, Oxford, 1965.

[12] Lifshitz, E. M. and Pitaevskii, L. P., Physical kinetics (English translation), Pergamon Press, Oxford, 1981.

[13] McKean, H. P., Propagation of chaos for a class of non-linear parabolic equations, Lecture series in differential equations, session 7: Catholic Univ., 1967.

[14] Murata, H., Propagation of chaos for Boltzmann-like equation of non-cutoff type in the plane, Hiroshima Math. J., 7 (1977), 479-515.

[15] Papanicolaou, G. C., Stroock, D. and Varadhan, S. R. S., Martingale approach to some limit theorems, 1976 Duke Turbulence Conf., Duke Univ. Math. Series III, 
1977.

[16] Skorohod, A. V., Limit theorems for Markov processes, Theor. Probability Appl., 3 (1957), 202-246.

[17] Spohn, H., Kinetic equations from Hamiltonian dynamics: Markovian limits, Rev. Modern Phys., 53 (1980), 569-615.

[18] Stroock, D. and Varadhan, S. R. S., Diffusion processes with continuous coefficients, I, II, Comm. Pure Appl. Math., 22 (1969), 345-400, 479-530.

[19] - Multidimensional diffusion processes, Springer-Verlag, Berlin 1979.

[20] Tanaka, H., Probabilistic treatment of the Boltzmann equation of Maxwellian molecules, Z. Wahrscheinlichkeitstheorie verw. Gebiete, 46 (1978), 67-105.

[21] - Stochastic differential equation associated with the Boltzmann equation of Maxwellian molecules in several dimensions, Stochastic Analysis (edited by Friedman and Pinsky), Academic Press, New York (1978), 301-314.

[22] — - Some probabilistic problems in the spatially homogeneous Boltzmann equation, Proc. of IFIP-ISI Conf. on Theory and Applications of Random Fields, Bangalore 1982. 